\title{
THE THOM ISOMORPHISM IN GAUGE-EQUIVARIANT $K$-THEORY
}

\author{
VICTOR NISTOR AND EVGENIJ TROITSKY
}

\begin{abstract}
In a previous paper [15], we have introduced the gauge-equivariant $K$-theory group $K_{\mathcal{G}}^{0}(X)$ of a bundle $\pi_{X}: X \rightarrow B$ endowed with a continuous action of a bundle of compact Lie groups $p: \mathcal{G} \rightarrow B$. These groups are the natural range for the analytic index of a family of gauge-invariant elliptic operators (i.e., a family of elliptic operators invariant with respect to the action of a bundle of compact groups). In this paper, we continue our study of gaugeequivariant $K$-theory. In particular, we introduce and study products, which helps us establish the Thom isomorphism in gauge-equivariant $K$-theory. Then we construct push-forward maps and define the topological index of a gaugeinvariant family.
\end{abstract}

\section{Contents}

1. Introduction 1

2. Preliminaries 3

2.1. Bundles of compact groups and finite holonomy conditions 3

2.2. Gauge-equivariant $K$-theory 4

2.3. Additional results 6

3. $K$-theory and complexes $\quad 8$

3.1. The $L_{\mathcal{G}}^{n}$-groups $\quad 8$

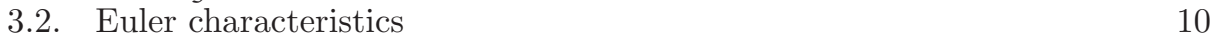

3.3. Globally defined complexes 14

3.4. The non-compact case $\quad 15$

4. The Thom isomorphism $\quad 15$

4.1. Products 16

4.2. The non-compact case $\quad 17$

5. Gysin maps 20

6. The topological index 24

References $\quad 29$

\section{INTRODUCTION}

In this paper we establish a Thom isomorphism theorem for gauge equivariant $K$-theory. Let $p: \mathcal{G} \rightarrow B$ be a bundle of compact groups. Recall that this means that each fiber $\mathcal{G}_{b}:=p^{-1}(b)$ is a compact group and that, locally, $\mathcal{G}$ is of the form

V. N. was partially supported by NSF Grants DMS-9971951, DMS 02-00808, and a "collaborative research" grant. E. T. was partially supported by RFFI Grant 05-01-00923, Grant for the support of leading scientific schools and Grant "Universities of Russia" YP.04.02.530. The present joint research was started under the hospitality of MPIM (Bonn). 
$U \times G$, where $U \subset B$ open and $G$ a fixed compact group. Let $X$ and $B$ be locally compact spaces and $\pi_{X}: X \rightarrow B$ be a continuous map. In the present paper, as in [15], this map will be supposed to be a locally trivial bundle. A part of present results can be extended to the case of a general map, this, as well as the proof of a general index theorem, will be the subject of a forthcoming paper.

Assume that $\mathcal{G}$ acts on $X$. This action will be always be fiber-preserving. Then we can associate to the action of $\mathcal{G}$ on $X \mathcal{G}$-equivariant $K$-theory groups $K_{\mathcal{G}}^{i}(X)$ as in [15]. We shall review and slightly generalize this definition in Section 2.

For $X$ compact, the group $K_{\mathcal{G}}^{0}(X)$ is defined as the Grothendieck group of $\mathcal{G}$-equivariant vector bundles on $X$. If $X$ is not compact, we define the groups $K_{\mathcal{G}}^{0}(X)$ using fiberwise one-point compactifications. We shall call these groups simply gauge-equivariant $K$-theory groups of $X$ when we do not want to specify $\mathcal{G}$. The reason for introducing the gauge-equivariant $K$-theory groups is that they are the natural range for the index of a gauge-invariant families of elliptic operators. In turn, the motivation for studying gauge-invariant families and their index is due to their connection to spectral theory and boundary value problems on non-compact manifolds. Some possible connections with Ramond-Ramond fields in String Theory were mentioned in $[8,15]$. See also $[1,9,12,13]$.

In this paper, we continue our study of gauge-equivariant $K$-theory. We begin by providing two alternative definitions of the relative $K_{\mathcal{G}}$-groups, both based on complexes of vector bundles. (In this paper, all vector bundles are complex vector bundles, with the exception of the tangent bundles and where explicitly stated.) These alternative definitions, modeled on the classical case $[2,10]$, provide a convenient framework for the study of products, especially in the relative or non-compact cases. The products are especially useful for the proof of the Thom isomorphism in gauge-equivariant theory, which is one of the main results of this paper. Let $E \rightarrow X$ be a $\mathcal{G}$-equivariant complex vector bundle. Then the Thom isomorphism is a natural isomorphism

$$
\tau_{E}: K_{\mathcal{G}}^{i}(X) \rightarrow K_{\mathcal{G}}^{i}(E) .
$$

(There is also a variant of this result for $\operatorname{spin}^{\mathrm{c}}$-vector bundles, but since we will not need it for the index theorem [14], we will not discuss this in this paper.) The Thom isomorphism allows us to define Gysin (or push-forward) maps in $K$-theory. As it is well known from the classical work of Atiyah and Singer [4], the Thom isomorphism and the Gysin maps are some of the main ingredients used for the definition and study of the topological index. In fact, we shall proceed along the lines of that paper to define the topological index for gauge-invariant families of elliptic operators. Some other approaches to Thom isomorphism in general settings of Noncommutative geometry were the subject of $[6,7,11,16,12]$ and many other papers.

Gauge-equivariant $K$-theory behaves in many ways like the usual equivariant $K$-theory, but exhibits also some new phenomena. For example, the groups $K_{\mathcal{G}}^{0}(B)$ may turn out to be reduced to $K^{0}(B)$ when $\mathcal{G}$ has "a lot of twisting" [15, Proposition 3.6]. This is never the case in equivariant $K$-theory when the action of the group is trivial but the group itself is not trivial. In [15], we addressed this problem in two ways: first, we found conditions on the bundle of groups $p: \mathcal{G} \rightarrow B$ that guarantee that $K_{\mathcal{G}}^{0}(X)$ is not too small (this condition is called finite holonomy and is recalled below), and, second, we studied a substitute of $K_{\mathcal{G}}^{0}(X)$ which is never too small (this 
substitute is $K\left(C^{*}(\mathcal{G})\right)$, the $K$-theory of the $C^{*}$-algebra of the bundle of compact groups $\mathcal{G})$.

In this paper, we shall again need the finite holonomy condition, so let us review it now. To define the finite holonomy condition, we introduced the representation covering of $\mathcal{G}$, denoted $\widehat{\mathcal{G}} \rightarrow B$. As a space, $\widehat{\mathcal{G}}$ is the union of all the representation spaces $\widehat{\mathcal{G}_{b}}$ of the fibers $\mathcal{G}_{b}$ of the bundle of compact groups $\mathcal{G}$. One measure of the twisting of the bundle $\mathcal{G}$ is the holonomy associated to the covering $\widehat{\mathcal{G}} \rightarrow B$. We say that $\mathcal{G}$ has representation theoretic finite holonomy if $\widehat{\mathcal{G}}$ is a union of compact-open subsets. (An equivalent conditions can be obtained in terms of the fundamental groups when $B$ is path-connected, see Proposition 2.3 below.)

Let $C^{*}(\mathcal{G})$ be the enveloping $C^{*}$-algebra of the bundle of compact groups $\mathcal{G}$. We proved in $[15$, Theorem 5.2$]$ that

$$
K_{\mathcal{G}}^{j}(B) \cong K_{j}\left(C^{*}(\mathcal{G})\right)
$$

provided that $\mathcal{G}$ has representation theoretic finite holonomy. This guarantees that $K_{\mathcal{G}}^{j}(B)$ is not too small. It also points out an alternative, algebraic definition of the groups $K_{\mathcal{G}}^{i}(X)$.

The structure of the paper is as follows. We start from the definition of gaugeequivariant $K$-theory and some basic results from [15], most of them related to the "finite holonomy condition," a condition on bundles of compact groups that we recall in Section 2. In Section 3 we describe an equivalent definition of gaugeequivariant $K$-theory in terms of complexes of vector bundles. This will turn out to be especially useful when studying the topological index. In Section 4 we establish the Thom isomorphism in gauge-equivariant $K$-theory, and, in Section 5 , we define and study the Gysin maps. The properties of the Gysin maps allow us to define in Section 6 the topological index and establish its main properties.

\section{Preliminaries}

We now recall the definition of gauge-equivariant $K$-theory and some basic results from [15]. An important part of our discussion is occupied by the discussion of the finite holonomy condition for a bundle of compact groups $p: \mathcal{G} \rightarrow B$.

All vector bundles in this paper are assumed to be complex vector bundles, unless otherwise mentioned and excluding the tangent bundles to the various manifolds appearing below.

2.1. Bundles of compact groups and finite holonomy conditions. We begin by introducing bundles of compact and locally compact groups. Then we study finite holonomy conditions for bundles of compact groups.

Definition 2.1. Let $B$ be a locally compact space and let $G$ be a locally compact group. We shall denote by $\operatorname{Aut}(G)$ the group of automorphisms of $G$. A bundle of locally compact groups $\mathcal{G}$ with typical fiber $G$ over $B$ is, by definition, a fiber bundle $\mathcal{G} \rightarrow B$ with typical fiber $G$ and structural group $\operatorname{Aut}(G)$.

We fix the above notation. Namely, from now on and throughout this paper, unless explicitly otherwise mentioned, $B$ will be a compact space and $\mathcal{G} \rightarrow B$ will be a bundle of compact groups with typical fiber $G$.

We need now to introduce the representation theoretic holonomy of a bundle of Lie group with compact fibers $p: \mathcal{G} \rightarrow B$. Let $\operatorname{Aut}(G)$ be the group of automorphisms of $G$. By definition, there exists then a principal Aut $(G)$-bundle $\mathcal{P} \rightarrow B$ 
such that

$$
\mathcal{G} \cong \mathcal{P} \times{ }_{\operatorname{Aut}(G)} G:=(\mathcal{P} \times G) / \operatorname{Aut}(G) .
$$

We shall fix $\mathcal{P}$ in what follows.

Let $\widehat{\mathcal{G}}$ be the (disjoint) union of the sets $\widehat{\mathcal{G}_{b}}$ of equivalence classes of irreducible representations of the groups $\mathcal{G}_{b}$. Using the natural action of $\operatorname{Aut}(G)$ on $\widehat{G}$, we can naturally identify $\widehat{\mathcal{G}}$ with $\mathcal{P} \times \operatorname{Aut}(G) \widehat{G}$ as fiber bundles over $B$.

Let $\operatorname{Aut}_{0}(G)$ be the connected component of the identity in $\operatorname{Aut}(G)$. The group $\operatorname{Aut}_{0}(G)$ will act trivially on the set $\widehat{G}$, because the later is discrete. Let

$$
H_{R}:=\operatorname{Aut}(G) / \operatorname{Aut}_{0}(G), \quad \mathcal{P}_{0}:=\mathcal{P} / \operatorname{Aut}_{0}(G), \quad \text { and } \hat{\mathcal{G}} \simeq \mathcal{P}_{0} \times_{H_{R}} \widehat{G} .
$$

Above, $\widehat{\mathcal{G}}$ is defined because $\mathcal{P}_{0}$ is an $H_{R}$-principal bundle. The space $\widehat{\mathcal{G}}$ will be called the representation space of $\mathcal{G}$ and the covering $\widehat{\mathcal{G}} \rightarrow B$ will be called the representation covering associated to $\mathcal{G}$.

Assume now that $B$ is a path-connected, locally simply-connected space and fix a point $b_{0} \in B$. We shall denote, as usual, by $\pi_{1}\left(B, b_{0}\right)$ the fundamental group of $B$. Then the bundle $\mathcal{P}_{0}$ is classified by a morphism

$$
\rho: \pi_{1}\left(B, b_{0}\right) \rightarrow H_{R}:=\operatorname{Aut}(G) / \operatorname{Aut}_{0}(G),
$$

which will be called the holonomy of the representation covering of $\mathcal{G}$.

For our further reasoning, we shall sometimes need the following finite holonomy condition.

Definition 2.2. We say that $\mathcal{G}$ has representation theoretic finite holonomy if every $\sigma \in \widehat{\mathcal{G}}$ is contained in a compact-open subset of $\widehat{\mathcal{G}}$.

In the cases we are interested in, the above condition can be reformulated as follows [15]

Proposition 2.3. Assume that $B$ is path-connected and locally simply-connected. Then $\mathcal{G}$ has representation theoretic finite holonomy if, and only if $\pi_{1}\left(B, b_{0}\right) \sigma \subset \widehat{G}$ is a finite set for any irreducible representation $\sigma$ of $G$.

From now on we shall assume that $\mathcal{G}$ has representation theoretic finite holonomy.

2.2. Gauge-equivariant $K$-theory. Let us now define the gauge equivariant $K$ theory groups of a "G-Giber bundle" $\pi_{Y}: Y \rightarrow B$. All our definitions are well known if $B$ is reduced to a point (cf. $[2,10]$ ). First we need to fix the notation.

If $f_{i}: Y_{i} \rightarrow B, i=1,2$, are two maps, we shall denote by

$$
Y_{1} \times_{B} Y_{2}:=\left\{\left(y_{1}, y_{2}\right) \in Y_{1} \times Y_{2}, f_{1}\left(y_{1}\right)=f_{2}\left(y_{2}\right)\right\}
$$

their fibered product. Let $p: \mathcal{G} \rightarrow B$ be a bundle of locally compact groups and let $\pi_{Y}: Y \rightarrow B$ be a continuous map. We shall say that $\mathcal{G}$ acts on $Y$ if each group $\mathcal{G}_{b}$ acts continuously on $Y_{b}:=\pi^{-1}(b)$ and the induced map $\mu$

$$
\mathcal{G} \times{ }_{B} Y:=\left\{(g, y) \in \mathcal{G} \times Y, p(g)=\pi_{Y}(y)\right\} \ni(g, y) \longrightarrow \mu(g, y):=g y \in Y
$$

is continuous. If $\mathcal{G}$ acts on $Y$, we shall say that $Y$ is a $\mathcal{G}$-space. If, in addition to that, $Y \rightarrow B$ is also locally trivial, we shall say that $Y$ is $a \mathcal{G}$-fiber bundle, or, simply, a $\mathcal{G}$-bundle. This definition is a particular case of the definition of the action of a differentiable groupoid on a space. 
Let $\pi_{Y}: Y \rightarrow B$ be a $\mathcal{G}$-space, with $\mathcal{G}$ a bundle of compact groups over $B$. Recall that a vector bundle $\tilde{\pi}_{E}: E \rightarrow Y$ is a $\mathcal{G}$-equivariant vector bundle (or simply a $\mathcal{G}$-equivariant vector bundle) if

$$
\pi_{E}:=\pi_{Y} \circ \tilde{\pi}_{E}: E \rightarrow B
$$

is a $\mathcal{G}$-space, the projection

$$
\tilde{\pi}_{E}: E_{b}:=\pi_{E}^{-1}(b) \rightarrow Y_{b}:=\pi_{Y}^{-1}(b)
$$

is $\mathcal{G}_{b}:=p^{-1}(b)$ equivariant, and the induced action $E_{y} \rightarrow E_{g y}$ of $g \in \mathcal{G}$, between the corresponding fibers of $E \rightarrow Y$, is linear for any $y \in Y_{b}, g \in \mathcal{G}_{b}$, and $b \in B$.

To define gauge-equivariant $K$-theory, we first recall some preliminary definitions from [15]. Let $\tilde{\pi}_{E}: E \rightarrow Y$ be a $\mathcal{G}$-equivariant vector bundle and let $\tilde{\pi}_{E^{\prime}}: E^{\prime} \rightarrow Y^{\prime}$ be a $\mathcal{G}^{\prime}$-equivariant vector bundle, for two bundles of compact groups $\mathcal{G} \rightarrow B$ and $\mathcal{G}^{\prime} \rightarrow B^{\prime}$. We shall say that $(\gamma, \varphi, \eta, \psi):\left(\mathcal{G}^{\prime}, E^{\prime}, Y^{\prime}, B^{\prime}\right) \rightarrow(\mathcal{G}, E, Y, B)$ is a $\gamma-$ equivariant morphism of vector bundles if the following five conditions are satisfied:

(i) $\gamma: \mathcal{G}^{\prime} \rightarrow \mathcal{G}, \varphi: E^{\prime} \rightarrow E, \eta: Y^{\prime} \rightarrow Y$, and $\psi: B \rightarrow B^{\prime}$,

(ii) all the resulting diagrams are commutative,

(iii) $\varphi(g e)=\gamma(g) \varphi(e)$ for all $e \in E_{b}^{\prime}$ and all $g \in \mathcal{G}_{b}^{\prime}$,

(iv) $\gamma$ is a group morphism in each fiber, and

(v) $f$ is a vector bundle morphism.

We shall say that $\phi: E \rightarrow E^{\prime}$ is a $\gamma$-equivariant morphism of vector bundles if, by definition, it is part of a morphism $(\gamma, \varphi, \eta, \psi):\left(\mathcal{G}^{\prime}, E^{\prime}, Y^{\prime}, B^{\prime}\right) \rightarrow(\mathcal{G}, E, Y, B)$. Note that $\eta$ and $\psi$ are determined by $\gamma$ and $\phi$.

As usual, if $\psi: B^{\prime} \rightarrow B$ is a continuous [respectively, smooth] map, we define the inverse image $\left(\psi^{*}(\mathcal{G}), \psi^{*}(E), \psi^{*}(Y), B^{\prime}\right)$ of a $\mathcal{G}$-equivariant vector bundle $E \rightarrow Y$ by $\psi^{*}(\mathcal{G})=\mathcal{G} \times{ }_{B} B^{\prime}, \psi^{*}(E)=E \times{ }_{B} B^{\prime}$, and $\psi^{*}(Y)=Y \times_{B} B^{\prime}$. If $B^{\prime} \subset B$ and $\psi$ is the embedding, this construction gives the restriction of a $\mathcal{G}$-equivariant vector bundle $E \rightarrow Y$ to a closed, invariant subset $B^{\prime} \subset B$ of the base of $\mathcal{G}$, yielding a $\mathcal{G}_{B^{\prime}}$-equivariant vector bundle. Usually $\mathcal{G}$ will be fixed, however.

Let $p: \mathcal{G} \rightarrow B$ be a bundle of compact groups and $\pi_{Y}: Y \rightarrow B$ be a $\mathcal{G}$-space. The set of isomorphism classes of $\mathcal{G}$-equivariant vector bundles $\tilde{\pi}_{E}: E \rightarrow Y$ will be denoted by $\mathcal{E}_{\mathcal{G}}(Y)$. On this set we introduce a monoid operation, denoted "+," using the direct sum of vector bundles. This defines a monoid structure on the set $\mathcal{E}_{\mathcal{G}}(Y)$ as in the case when $B$ consists of a point.

Definition 2.4. Let $\mathcal{G} \rightarrow B$ be a bundle of compact groups acting on the $\mathcal{G}$-space $Y \rightarrow B$. Assume $Y$ to be compact. The $\mathcal{G}$-equivariant $K$-theory group $K_{\mathcal{G}}^{0}(Y)$ is defined as the group completion of the monoid $\mathcal{E}_{\mathcal{G}}(Y)$.

When working with gauge-equivariant $K$-theory, we shall use the following terminology and notation. If $E \rightarrow Y$ is a $\mathcal{G}$-equivariant vector bundle on $Y$, we shall denote by $[E]$ its class in $K_{\mathcal{G}}^{0}(Y)$. Thus $K_{\mathcal{G}}^{0}(Y)$ consists of differences $[E]-\left[E^{1}\right]$. The groups $K_{\mathcal{G}}^{0}(Y)$ will also be called gauge equivariant $K$-theory groups, when we do not need to specify $\mathcal{G}$. If $B$ is reduced to a point, then $\mathcal{G}$ is group, and the groups $K_{\mathcal{G}}^{0}(Y)$ reduce to the usual equivariant $K$-groups.

We have the following simple observations on gauge-equivariant $K$-theory. First, the familiar functoriality properties of the usual equivariant $K$-theory groups extend to the gauge equivariant $K$-theory groups. For example, assume that the bundle of compact groups $\mathcal{G} \rightarrow B$ acts on a fiber bundle $Y \rightarrow B$ and that, similarly, $\mathcal{G}^{\prime} \rightarrow B^{\prime}$ acts on a fiber bundle $Y^{\prime} \rightarrow B^{\prime}$. Let $\gamma: \mathcal{G} \rightarrow \mathcal{G}^{\prime}$ be a morphism of bundles of 
compact groups and $f: Y \rightarrow Y^{\prime}$ be a $\gamma$-equivariant map. Then we obtain a natural group morphism

$$
(\gamma, f)^{*}: K_{\mathcal{G}^{\prime}}^{0}\left(Y^{\prime}\right) \rightarrow K_{\mathcal{G}}^{0}(Y) .
$$

If $\gamma$ is the identity morphism, we shall denote $(\gamma, f)^{*}=f^{*}$.

A $\mathcal{G}$-equivariant vector bundle $E \rightarrow Y$ on a $\mathcal{G}$-space $Y \rightarrow B, Y$ compact, is called trivial if, by definition, there exists a $\mathcal{G}$-equivariant vector bundle $E^{\prime} \rightarrow B$ such that $E$ is isomorphic to the pull-back of $E^{\prime}$ to $Y$. Thus $E \simeq Y \times_{B} E^{\prime}$. If $\mathcal{G} \rightarrow B$ has representation theoretic finite holonomy and $Y$ is a compact $\mathcal{G}$-bundle, then every $\mathcal{G}$-equivariant vector bundle over $Y$ can be embedded into a trivial $\mathcal{G}$-equivariant vector bundle. This embedding will necessarily be as a direct summand.

If $\mathcal{G} \rightarrow B$ does not have finite holonomy, it is possible to provide examples if $\mathcal{G}$-equivariant vector bundles that do not embed into trivial $\mathcal{G}$-equivariant vector bundles [15]. Also, a related example from [15] shows that the groups $K_{\mathcal{G}}^{0}(Y)$ can be fairly small if the holonomy of $\mathcal{G}$ is "large."

A further observation is that it follows from the definition that the tensor product of vector bundles defines a natural ring structure on $K_{\mathcal{G}}^{0}(Y)$. We shall denote the product of two elements $a$ and $b$ in this ring by $a \otimes b$ or, simply, $a b$, when there is no danger of confusion. In particular the groups $K_{\mathcal{G}}^{i}(X)$ for $\pi_{X}: X \rightarrow B$ are equipped with a natural structure of $K_{\mathcal{G}}^{0}(B)$-module obtained using the pull-back of vector bundles on $B$, namely, $a b:=\pi_{X}^{*}(a) \otimes b \in K_{\mathcal{G}}^{0}(X)$ for $a \in K_{\mathcal{G}}^{0}(B)$ and $b \in K_{\mathcal{G}}^{0}(X)$.

The definition of the gauge-equivariant groups extends to non-compact $\mathcal{G}$-spaces $Y$ as in the case of equivariant $K$-theory. Let $Y$ be a $\mathcal{G}$-bundle. We shall denote then by $Y^{+}:=Y \cup B$ the compact space obtained from $Y$ by the one-point compactification of each fiber (recall that $B$ is compact). The need to consider the space $Y^{+}$is the main reason for considering also non longitudinally smooth fibers bundles on $B$. Then

$$
K_{\mathcal{G}}^{0}(Y):=\operatorname{ker}\left(K_{\mathcal{G}}^{0}\left(Y^{+}\right) \rightarrow K_{\mathcal{G}}^{0}(B)\right) .
$$

Also as in the classical case, we let

$$
K_{\mathcal{G}}^{n}\left(Y, Y^{\prime}\right):=K_{\mathcal{G}}^{0}\left(\left(Y \backslash Y^{\prime}\right) \times \mathbb{R}^{n}\right)
$$

for a $\mathcal{G}$-subbundle $Y^{\prime} \subset Y$. Then [15] we have the following periodicity result

Theorem 2.5. We have natural isomorphisms

$$
K_{\mathcal{G}}^{n}\left(Y, Y^{\prime}\right) \cong K_{\mathcal{G}}^{n-2}\left(Y, Y^{\prime}\right) .
$$

Gauge-equivariant $K$-theory is functorial with respect to open embeddings. Indeed, let $U \subset X$ be an open, $\mathcal{G}$-equivariant subbundle. Then the results of $[15$, Section 3] provide us with a natural map morphism

$$
i_{*}: K_{\mathcal{G}}^{n}(U) \rightarrow K_{\mathcal{G}}^{n}(X) .
$$

In fact, $i_{*}$ is nothing but the composition $K_{\mathcal{G}}^{n}(U) \cong K_{\mathcal{G}}^{n}(X, X \backslash U) \rightarrow K_{\mathcal{G}}^{n}(X)$.

2.3. Additional results. We now prove some more results on gauge-equivariant $K$-theory.

Let $\mathcal{G} \rightarrow B$ and $\mathcal{H} \rightarrow B$ be two bundles of compact groups over $B$. Recall that an $\mathcal{H}$-bundle $\pi_{X}: X \rightarrow B$ is called free if the action of each group $\mathcal{H}_{b}$ on the fiber $X_{b}$ is free (i.e., $h x=x, x \in X_{b}$, implies that $h$ is the identity of $\mathcal{G}_{b}$.) We shall need the following result, which is an extension of a result in [10, page 69]. For simplicity, we shall write $\mathcal{G} \times \mathcal{H}$ instead of $\mathcal{G} \times_{B} \mathcal{H}$. 
Theorem 2.6. Suppose $\pi_{X}: X \rightarrow B$ is a $\mathcal{G} \times \mathcal{H}$-bundle that is free as an $\mathcal{H}$ bundle. Let $\pi: X \rightarrow X / \mathcal{H}$ be the (fiberwise) quotient map. For any $\mathcal{G}$-equivariant vector bundle $\tilde{\pi}_{E}: E \rightarrow X / \mathcal{H}$, we define the induced vector bundle

$$
\pi^{*}(E):=\left\{(x, \varepsilon) \in X \times E, \pi(x)=\tilde{\pi}_{E}(\varepsilon)\right\} \rightarrow X,
$$

with the action of $\mathcal{G} \times \mathcal{H}$ given by $(g, h) \cdot(x, \varepsilon):=((g, h) x, g \varepsilon)$. Then $\pi^{*}$ is gives rise to a natural isomorphism $K_{\mathcal{G}}^{0}(X / \mathcal{H}) \rightarrow K_{\mathcal{G} \times \mathcal{H}}^{0}(X)$.

Proof. Let $\pi^{*}: K_{\mathcal{G}}^{0}(X / \mathcal{H}) \rightarrow K_{\mathcal{G} \times \mathcal{H}}^{0}(X)$ be the induction map, as above. We will construct a map $r: K_{\mathcal{G} \times \mathcal{H}}^{0}(X) \rightarrow K_{\mathcal{G}}^{0}(X / \mathcal{H})$ satisfying $\pi^{*} \circ r=\mathrm{Id}$ and $r \circ \pi^{*}=\mathrm{Id}$. Let $\pi_{F}: F \rightarrow X$ be a $\mathcal{G} \times \mathcal{H}$-vector bundle. Since the action of $\mathcal{H}$ on $X$ is free, the induced map $\bar{\pi}_{F}: F / \mathcal{H} \rightarrow X / \mathcal{H}$ of quotient spaces is a (locally trivial) $\mathcal{G}$-bundle. Clearly, this construction is invariant under homotopy, and hence we can define $r[F]:=[F / \mathcal{H}]$.

Let us check now that $r$ is indeed an inverse of $\pi^{*}$. Denote by $F \ni f \rightarrow \mathcal{H} f \in$ $F / \mathcal{H}$ the quotient map. Let $F \rightarrow X$ be a $\mathcal{G} \times \mathcal{H}$-vector bundle. To begin with, the total space of $\pi^{*} \circ r(F)$ is

$$
\left\{(x, \mathcal{H} f) \in X \times(F / \mathcal{H}), \pi^{*}(x)=\bar{\pi}_{F}(\mathcal{H} f)\right\},
$$

by definition. Then the map $F \ni f \rightarrow\left(\pi_{F}(f), \mathcal{H} f\right) \in \pi^{*} \circ r(F)$ is an isomorphism. Hence, $\pi^{*} r=\mathrm{Id}$.

Next, consider a $\mathcal{G}$-vector bundle $\pi_{E}: E \rightarrow X / \mathcal{H}$. The total space of $r \circ \pi^{*}(E)$ is then

$$
\left.\left\{(\mathcal{H} y, \varepsilon) \in(X / \mathcal{H}) \times E, \mathcal{H} y=\pi_{E}(\varepsilon)\right)\right\},
$$

because $\mathcal{H}$ acts only on the first component of $\pi^{*}(E)$. Then

$$
E \rightarrow r \circ \pi^{*}(E), \quad \varepsilon \mapsto(\pi(\varepsilon), \varepsilon)
$$

is an isomorphism. Hence, $r \circ \pi^{*}=\mathrm{Id}$.

See also [15, Theorem 3.5].

Corollary 2.7. Let $P$ be $a \mathcal{G} \times \mathcal{H}$-bundle that is free as an $\mathcal{H}$-bundle. Also, let $W$ be an $\mathcal{H}$-bundle, then there is a natural isomorphism

$$
K_{\mathcal{G} \times \mathcal{H}}\left(P \times_{B} W\right) \cong K_{\mathcal{G}}\left(P \times_{\mathcal{H}} W\right) .
$$

Proof. Take $X:=P \times_{B} W$ in the previous theorem. Then $X$ is a free $\mathcal{H}$-bundle, because $P$ is, and $X / \mathcal{H}=: P \times_{\mathcal{H}} W$.

In the following section, we shall also need the following quotient construction associated to a trivialization of a vector bundle over a subset. Namely, if $Y \subset X$ is a $\mathcal{G}$-invariant, closed subbundle, then we shall denote by $X /{ }_{B} Y$ the fiberwise quotient space over $B$, that is the quotient of $X$ with respect to the equivalence relation $\sim, x \sim y$ if, and only if, $x, y \in Y_{b}$, for some $b \in B$.

If $E$ is a $\mathcal{G}$-equivariant vector bundle over a $\mathcal{G}$-bundle $X$, together with a $\mathcal{G}$ equivariant trivialization over a $\mathcal{G}$-subbundle $Y \subset X$, then we can generalize the quotient (or collapsing) construction of $[2, \S 1.4]$ to obtain a vector bundle over $X /{ }_{B} Y$, where by $X /{ }_{B} Y$ we denote the fiberwise quotient bundle over $B$, as above.

Lemma 2.8. Suppose that $X$ is a $\mathcal{G}$-bundle and that $Y \subset X$ is a closed, $\mathcal{G}$ invariant subbundle. Let $E \rightarrow X$ be a $\mathcal{G}$-vector bundle and $\alpha:\left.E\right|_{Y} \cong Y \times_{B} \mathcal{V}$ be a $\mathcal{G}$-equivariant trivialization, where $\mathcal{V} \rightarrow B$ is a $\mathcal{G}$-equivariant vector bundle. Then 
we can naturally associate to $(E, \alpha)$ a naturally defined vector bundle $E / \alpha \rightarrow X /{ }_{B} Y$ that depends only on homotopy class of $\alpha$.

Proof. Let $p: Y \times{ }_{B} \mathcal{V} \rightarrow \mathcal{V}$ be the natural projection. Introduce the following equivalence relation on $E$ :

$$
e \sim e^{\prime} \Leftrightarrow e,\left.e^{\prime} \in E\right|_{Y} \text { and } p \alpha(e)=p \alpha\left(e^{\prime}\right) .
$$

Let then $E / \alpha$ be equal to $E / \sim$. This is locally trivial vector bundle over $X /{ }_{B} Y$. Indeed, it is necessary to verify this only in a neighborhood of $Y /{ }_{B} Y \cong B$. Let $U$ be a $\mathcal{G}$ invariant open subset of $X$ such that $\alpha$ can be extended to an isomorphism $\widetilde{\alpha}:\left.E\right|_{U} \cong U \times_{B} \mathcal{V}$. We obtain an isomorphism

$$
\alpha^{\prime}:\left(\left.E\right|_{U}\right) / \alpha \cong\left(U /{ }_{B} Y\right) \times_{B} \mathcal{V}, \quad \alpha^{\prime}(e)=\widetilde{\alpha}(e)
$$

Moreover, $\left(U /{ }_{B} Y\right) \times{ }_{B} \mathcal{V}$ is a locally trivial $\mathcal{G}$-equivariant vector bundle.

Suppose that $\alpha_{0}$ and $\alpha_{1}$ are homotopic trivializations of $\left.E\right|_{Y}$, that is, trivializations such that there exists a trivialization $\beta: E \times\left. I\right|_{Y \times I} \cong Y \times I \times{ }_{B} \mathcal{V}$, $\left.\beta\right|_{E \times\{0\}}=\alpha_{0}$ and $\left.\beta\right|_{E \times\{1\}}=\alpha_{1}$. Let

$$
f: X /{ }_{B} Y \times I \rightarrow(X \times I) /{ }_{(B \times I)}(Y \times I) .
$$

Then the bundle $f^{*}((E \times I) / \beta)$ over $X /{ }_{B} Y \times I$ satisfies $\left.f^{*}((E \times I) / \beta)\right|_{\left(X /{ }_{B} Y\right) \times\{i\}}=$ $E / \alpha_{i}, i=0,1$. Hence, $E / \alpha_{0} \cong E / \alpha_{1}$.

\section{3. $K$-THEORY AND COMPLEXES}

For the purpose of defining the Thom isomorphism, it is convenient to work with an equivalent definition of gauge-equivariant $K$-theory in terms of complexes of vector bundles. This will turn out to be especially useful when studying the topological index.

The statements and proofs of this section, except maybe Lemma 3.4, follow the classical ones $[2,10]$, so our presentation will be brief.

3.1. The $L_{\mathcal{G}}^{n}$-groups. We begin by adapting some well known concepts and constructions to our settings.

Let $X \rightarrow B$ be a locally compact, paracompact $\mathcal{G}$-bundle. A finite complex of $\mathcal{G}$-equivariant vector bundles over $X$ is a complex

$$
\left(E^{*}, d\right)=\left(\ldots \stackrel{d_{i-1}}{\longrightarrow} E^{i} \stackrel{d_{i}}{\longrightarrow} E^{i+1} \stackrel{d_{i+1}}{\longrightarrow} \ldots\right)
$$

$i \in \mathbb{Z}$, of $\mathcal{G}$-equivariant vector bundles over $X$ with only finitely many $E^{i}$ 's different from zero. Explicitly, $E^{i}$ are $\mathcal{G}$-equivariant vector bundles, $d_{i}$ 's are $\mathcal{G}$-equivariant morphisms, $d_{i+1} d_{i}=0$ for every $i$, and $E^{i}=0$ for $|i|$ large enough. We shall also use the notation $\left(E^{*}, d\right)=\left(E^{0}, \ldots, E^{n}, d_{i}:\left.\left.E^{i}\right|_{Y} \rightarrow E^{i+1}\right|_{Y}\right)$, if $E^{i}=0$ for $i<0$ and for $i>n$.

As usual, a morphism of complexes $f:\left(E^{*}, d\right) \rightarrow\left(F^{*}, \delta\right)$ is a sequence of morphisms $f_{i}: E^{i} \rightarrow F^{i}$ such that $f_{i+1} d_{i}=\delta_{i+1} f_{i}$, for all $i$. These constructions yield the category of finite complexes of $\mathcal{G}$-equivariant vector bundles. Isomorphism in this category will be denoted by $\left(E^{*}, d\right) \cong\left(F^{*}, \delta\right)$.

In what follows, we shall consider a pair $(X, Y)$ of $\mathcal{G}$-bundles with $X$ is a compact $\mathcal{G}$-bundle, unless explicitly otherwise mentioned. 
Definition 3.1. Let $X$ be a compact $\mathcal{G}$-bundle and $Y$ be a closed $\mathcal{G}$-invariant subbundle. Denote by $C_{\mathcal{G}}^{n}(X, Y)$ the set of (isomorphism classes of) sequences

$$
\left(E^{*}, d\right)=\left(E^{0}, E^{1}, \ldots, E^{n}, d_{k}:\left.\left.E^{k}\right|_{Y} \rightarrow E^{k+1}\right|_{Y}\right)
$$

of $\mathcal{G}$-equivariant vector bundles over $X$ such that $\left(\left.E^{k}\right|_{Y}, d\right)$ is exact if we let $E^{j}=0$ for $j<0$ or $j>n$.

We endow $C_{\mathcal{G}}^{n}(X, Y)$ with the semigroup structure given by the direct sums of complexes. An element in $C_{\mathcal{G}}^{n}(X, Y)$ is called elementary if it is isomorphic to a complex of the form

$$
\ldots \rightarrow 0 \rightarrow E \stackrel{\mathrm{Id}}{\longrightarrow} E \rightarrow 0 \rightarrow \ldots,
$$

Two complexes $\left(E^{*}, d\right),\left(F^{*}, \delta\right) \in C_{\mathcal{G}}^{n}(X, Y)$ are called equivalent if, and only if, there exist elementary complexes $Q^{1}, \ldots, Q^{k}, P^{1}, \ldots, P^{m} \in C_{\mathcal{G}}^{n}(X, Y)$ such that

$$
E \oplus Q_{1} \oplus \cdots \oplus Q_{k} \cong F \oplus P_{1} \oplus \cdots \oplus P_{m} .
$$

We write $E \simeq F$ in this case. The semigroup of equivalence classes of sequences in $C_{\mathcal{G}}^{n}(X, Y)$ will be denoted by $L_{\mathcal{G}}^{n}(X, Y)$.

We obtain, from definition, natural injective semigroup homomorphisms

$$
C_{\mathcal{G}}^{n}(X, Y) \rightarrow C_{\mathcal{G}}^{n+1}(X, Y) \text { and } C_{\mathcal{G}}(X, Y):=\bigcup_{n} C_{\mathcal{G}}^{n}(X, Y)
$$

The equivalence relation $\sim$ commutes with embeddings, so the above morphisms induce morphisms $L_{\mathcal{G}}^{n}(X, Y) \rightarrow L_{\mathcal{G}}^{n+1}(X, Y)$. Let $L_{\mathcal{G}}^{\infty}(X, Y):=\lim _{\rightarrow} L_{\mathcal{G}}^{n}(X, Y)$.

Lemma 3.2. Let $E \rightarrow X$ and $F \rightarrow X$ be $\mathcal{G}$-vector bundles. Let $\alpha:\left.\left.E\right|_{Y} \rightarrow F\right|_{Y}$ and $\beta: E \rightarrow F$ be surjective morphisms of $\mathcal{G}$-equivariant vector bundles. Also, assume that $\alpha$ and $\left.\beta\right|_{Y}$ are homotopic in the set of surjective $\mathcal{G}$-equivariant vector bundle morphisms. Then there exists a surjective morphism of $\mathcal{G}$-equivariant vector bundles $\widetilde{\alpha}: E \rightarrow F$ such that $\left.\widetilde{\alpha}\right|_{Y}=\alpha$. The same result remains true if we replace "surjective" with "injective" or "isomorphism" everywhere.

Proof. Let $Z:=(Y \times[0,1]) \cup(X \times\{0\})$ and $\pi: Z \rightarrow X$ be the projection. Let $\pi^{*}(E) \rightarrow Z$ and $\pi^{*}(F) \rightarrow Z$ be the pull-backs of $E$ and $F$. The homotopy in the statement of the Lemma defines a surjective morphism $a: \pi^{*}(E) \rightarrow \pi^{*}(F)$ such that $\left.a\right|_{Y \times\{1\}}=\alpha$ and $\left.a\right|_{X \times\{0\}}=\beta$. By [15, Lemma 3.12], the morphism $a$ can be extended to a surjective morphism over $(U \times[0,1]) \cup(X \times\{0\})$, where $U$ is an open $\mathcal{G}$-neighborhood of $Y$. (In fact, in that Lemma we considered only the case of an isomorphism, but the case of a surjective morphism is proved in the same way.) Let $\varphi: X \rightarrow[0,1]$ be a continuous function such that $\varphi(Y)=1$ and $\varphi(X \backslash U)=0$. By averaging, we can assume $\varphi$ to be $\mathcal{G}$-equivariant. Then define $\widetilde{\alpha}(x)=a(x, \varphi(x))$, for all $x \in X$.

Remark 3.3. Suppose that $X$ is a compact $\mathcal{G}$-space and $Y=\emptyset$. Then we have a natural isomorphism $\chi_{1}: L_{\mathcal{G}}^{1}(X, \emptyset) \rightarrow K_{\mathcal{G}}^{0}(X)$ taking the class of $E^{1}, E^{0}$ to the element $\left[E^{0}\right]-\left[E^{1}\right]$.

We shall need the following lemma.

Lemma 3.4. Let $p: \mathcal{G} \rightarrow B$ be a bundle of compact groups and $\pi_{X}: X \rightarrow B$ be a compact $\mathcal{G}$-bundle. Assume that $\pi_{X}$ has a cross-section, which we shall use to identify $B$ with a subset of $X$. Then the sequence

$$
0 \rightarrow L_{\mathcal{G}}^{1}(X, B) \rightarrow L_{\mathcal{G}}^{1}(X) \rightarrow L_{\mathcal{G}}^{1}(B)
$$


is exact.

Proof. Suppose that $E=\left(E^{1}, E^{0}, \varphi\right)$ defines an element of $L_{\mathcal{G}}^{1}(X)$ such that its image in $L_{\mathcal{G}}^{1}(B)$ is zero. Then the definition of $E \sim 0$ in $L_{\mathcal{G}}^{1}(B)$ shows that the restrictions of $E^{1}$ and $E^{0}$ to $B$ are isomorphic over $B$. Hence, the above sequence is exact at $L_{\mathcal{G}}^{1}(X)$.

Suppose now that $\left(E^{1}, E^{0}, \varphi\right)$ represents a class in $L_{\mathcal{G}}^{1}(X, B)$ such that its image in $L_{\mathcal{G}}^{1}(X)$ is zero. This means (keeping in mind Remark 3.3) that there exists a $\mathcal{G}-$ equivariant vector bundle $\widetilde{P}$ and an isomorphism $\widetilde{\psi}: E^{1} \oplus \widetilde{P} \cong E^{0} \oplus \widetilde{P}$. Let us define $P:=\widetilde{P} \oplus \pi_{X}^{*}\left(\left.E^{0}\right|_{B}\right) \oplus \pi_{X}^{*}\left(\left.\widetilde{P}\right|_{B}\right)$, where $\pi_{X}: X \rightarrow B$ is the canonical projection, as in the statement of the Lemma. Also, define $\psi=\widetilde{\psi} \oplus \mathrm{Id}: E^{1} \oplus P \rightarrow E^{0} \oplus P$, which is also an isomorphism.

We thus obtain that $T:=\psi(\varphi \oplus \mathrm{Id})^{-1}$ is an automorphism of $\left.\left(E^{0} \oplus P\right)\right|_{B}$. which has the form $\left(\begin{array}{cc}\beta & 0 \\ 0 & \text { Id }\end{array}\right)$ with the respect to the decomposition

$$
\left.\left(E^{0} \oplus P\right)\right|_{B}=\left.\left.\left(E^{0} \oplus \widetilde{P}\right)\right|_{B} \oplus\left(E^{0} \oplus \widetilde{P}\right)\right|_{B} .
$$

The automorphism $T:=\psi(\varphi \oplus \mathrm{Id})^{-1}$ is homotopic to the automorphism $T_{1}$ defined by the matrix

$$
\left(\begin{array}{cc}
\operatorname{Id} & 0 \\
0 & \beta
\end{array}\right) \text {. }
$$

Since $T_{1}$ extends to an automorphism of $E^{0} \oplus P$ over $X$, namely $\left(\begin{array}{cc}\operatorname{Id} & 0 \\ 0 & \pi_{X}^{*}(\beta)\end{array}\right)$, Lemma 3.2 gives that the automorphism $\psi(\varphi \oplus \mathrm{Id})^{-1}$ also can be extended to $X$, such that over $B$ we have the following commutative diagram:

$$
\begin{aligned}
& \left.\left.\left(E^{1} \oplus P\right)\right|_{B} \stackrel{\varphi \oplus \mathrm{Id}}{\longrightarrow}\left(E^{0} \oplus P\right)\right|_{B} \\
& \left.\downarrow \psi\right|_{B} \\
& \left.\left.\left(E^{0} \oplus P\right)\right|_{B} \stackrel{\text { Id }}{\longrightarrow}\left(E^{0} \oplus P\right)\right|_{B}=\left(\begin{array}{cc}
\beta & 0 \\
0 & \text { Id }
\end{array}\right)
\end{aligned}
$$

Hence, $\left(E^{1}, E^{0}, \varphi\right) \oplus(P, P, \mathrm{Id}) \cong\left(E^{0} \oplus P, E^{0} \oplus P, \mathrm{Id}\right)$ and so is zero in $L_{\mathcal{G}}^{1}(X, B)$.

3.2. Euler characteristics. We now generalize the above construction to other groups $L_{\mathcal{G}}^{n}$, thus proving the existence and uniqueness of Euler characteristics.

Definition 3.5. Let $X$ be a compact $\mathcal{G}$-space and $Y \subset X$ be a $\mathcal{G}$-invariant subset. An Euler characteristic $\chi_{n}$ is a natural transformation of functors $\chi_{n}: L_{\mathcal{G}}^{n}(X, Y) \rightarrow$ $K_{\mathcal{G}}^{0}(X, Y)$, such that for $Y=\emptyset$ it takes the form

$$
\chi_{n}(E)=\sum_{i=0}^{n}(-1)^{i}\left[E^{i}\right],
$$

for any sequence $E=\left(E^{*}, d\right) \in L_{\mathcal{G}}^{n}(X, Y)$.

Lemma 3.6. There exists a unique natural transformation of functors (i.e., an Euler characteristic)

$$
\chi_{1}: L_{\mathcal{G}}^{1}(X, Y) \rightarrow K_{\mathcal{G}}^{0}(X, Y),
$$

which, for $Y=\emptyset$, has the form indicated in 3.3 . 
Proof. To prove the uniqueness, suppose that $\chi_{1}$ and $\chi_{1}^{\prime}$ are two Euler characteristics on $L_{\mathcal{G}}^{1}$. Then $\chi_{1}^{\prime} \chi_{1}^{-1}$ is a natural transformation of $K_{\mathcal{G}}^{0}$ that is equal to the identity on each $K_{\mathcal{G}}^{0}(X)$. Let us consider the long exact sequence a long exact sequence

$$
\begin{aligned}
\cdots \rightarrow K_{\mathcal{G}}^{n-1}\left(Y, Y^{\prime}\right) \rightarrow K_{\mathcal{G}}^{n-1}(Y) & \rightarrow K_{\mathcal{G}_{1}}^{n-1}\left(Y^{\prime}\right) \\
& \rightarrow K_{\mathcal{G}}^{n}\left(Y, Y^{\prime}\right) \rightarrow K_{\mathcal{G}}^{n}(Y) \rightarrow K_{\mathcal{G}}^{n}\left(Y^{\prime}\right) \rightarrow \ldots
\end{aligned}
$$

associated to a pair $(Y, Y)$ of $\mathcal{G}$-bundles (see [15], Equation (10) for a proof of the exactness of this sequence). The map $K_{\mathcal{G}}^{0}(X, B) \rightarrow K_{\mathcal{G}}^{0}(X)$ from this exact sequence is induced by $(X, \emptyset) \rightarrow(X, B)$, and hence, in particular, it is natural. Assume that $\pi_{X}: X \rightarrow B$ has a cross-section. Then the exact sequence (7) for $\left(Y, Y^{\prime}\right)=(X, B)$ yields a natural exact sequence $0 \rightarrow K_{\mathcal{G}}^{0}(X, B) \rightarrow K_{\mathcal{G}}^{0}(X)$. This in conjunction with Lemma 3.4 shows that $\chi_{1}^{\prime} \chi_{1}^{-1}$ is the identity on $K_{\mathcal{G}}^{0}(X, B)$. Recall now that we agreed to denote by $X /{ }_{B} Y$ the fiberwise quotient space over $B$, that is the quotient of $X$ with respect to the equivalence relation $\sim, x \sim y$ if, and only if, $x, y \in Y_{b}$, for some $b \in B$. Finally, since the map $(X, Y) \rightarrow\left(X /{ }_{B} Y, B\right)$ induces an isomorphism of $K_{\mathcal{G}}^{0}$-groups [15, Theorem 3.19], $\chi_{1}^{\prime} \chi_{1}^{-1}$ is the identity on $K_{\mathcal{G}}^{0}(X, Y)$ for all pairs $(X, Y)$.

To prove the existence of the Euler characteristic $\chi_{1}$, let $\left(E^{1}, E^{0}, \alpha\right):=(\alpha$ : $\left.E^{1} \rightarrow E^{0}\right)$ represent an element of $L_{\mathcal{G}}^{1}(X, Y)$. Suppose that $X_{0}$ and $X_{1}$ are two copies of $X$ and $Z:=X_{0} \cup_{Y} X_{1} \rightarrow B$ is the $\mathcal{G}$-bundle obtained by identifying the two copies of $Y \subset X_{i}, i=0,1$. The identification of $\left.E^{1}\right|_{Y}$ and $\left.E^{0}\right|_{Y}$ with the help of $\alpha$ gives rise to an element $\left[F^{0}\right]-\left[F^{1}\right] \in K_{\mathcal{G}}^{0}(Z)$ defined as follows. By adding some bundle to both $E^{i}$ 's, we can assume that $E^{1}$ is trivial (that is, it is isomorphic to the pull-back of a vector bundle on $B$ ). Then $E^{1}$ extends to a trivial $\mathcal{G}$-vector bundle $\widetilde{E}^{1} \rightarrow Z$. We define $F^{0}:=E^{0} \cup_{\alpha} E^{1}$ and $F^{1}:=\widetilde{E}^{1}$.

The exact sequence $(7)$ and the natural $\mathcal{G}$-retractions $\pi_{i}: Z \rightarrow X_{i}$, give natural direct sum decompositions

$$
K_{\mathcal{G}}^{0}(Z)=K_{\mathcal{G}}^{0}\left(Z, X_{i}\right) \oplus K_{\mathcal{G}}^{0}\left(X_{i}\right), \quad i=0,1 .
$$

The natural map $\left(X_{0}, Y\right) \rightarrow\left(Z, X_{1}\right)$ induces an isomorphism

$$
k: K_{\mathcal{G}}^{0}\left(Z, X_{1}\right) \rightarrow K_{\mathcal{G}}^{0}\left(X_{0}, Y\right) .
$$

Let us define then $\chi_{1}\left(E^{0}, E^{1}, \alpha\right)$ to be equal to the image under $k$ of the $K_{\mathcal{G}}^{0}\left(Z, X_{1}\right)$ component of $\left[E^{1}, \alpha, E^{0}\right]$ (with the respect to (8)). It follows from its definition that this map is natural, respects direct sums, and is independent with respect to the addition of elementary elements. Our proof is completed by observing that $\chi_{1}\left(E^{1}, E^{0}, \alpha\right)=\left[E^{0}\right]-\left[E^{1}\right]$ when $Y=\emptyset$.

We shall also need the following continuity property of the functor $L_{\mathcal{G}}^{1}$. Recall that we have agreed to denote by $X /{ }_{B} Y$ the fiberwise quotient bundle over $B$.

Lemma 3.7. The natural homomorphism

$$
\Pi^{*}: L_{\mathcal{G}}^{1}\left(X /{ }_{B} Y, Y /{ }_{B} Y\right)=L_{\mathcal{G}}^{1}\left(X /{ }_{B} Y, B\right) \rightarrow L_{\mathcal{G}}^{1}(X, Y)
$$

is an isomorphism for all pairs $(X, Y)$ of compact $\mathcal{G}$-bundles. 
Proof. Lemmata 3.6 and 3.4 give the following commutative diagram

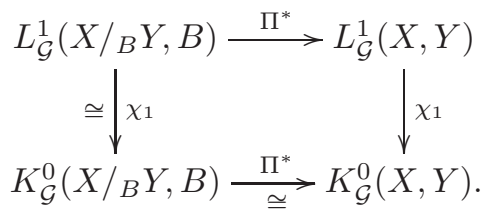

From this we obtain injectivity.

To prove surjectivity, suppose that $E^{1}$ and $E^{0}$ are $\mathcal{G}$-equivariant vector bundles over $X$ and $\alpha:\left.\left.E^{1}\right|_{Y} \rightarrow E^{0}\right|_{Y}$ is an isomorphism of the restrictions. Let $P \rightarrow X$ be a $\mathcal{G}$-bundle such that there is an isomorphism $\beta: E^{1} \oplus P \cong F$, where $F$ is a trivial bundle (i.e., isomorphic to a pull back from $B$ ). Then $\left(E^{1}, E^{0}, \alpha\right) \sim\left(F, E^{0} \oplus P, \gamma\right)$, where $\gamma=(\alpha \oplus \operatorname{Id}) \beta^{-1}$. The last object is the image of $\left(F,\left(E^{0} \oplus P\right) / \gamma, \gamma / \gamma\right)$ (see Lemma 2.8).

We obtain the following corollaries.

Corollary 3.8. The Euler characteristic $\chi_{1}: L_{\mathcal{G}}^{1}(X, Y) \rightarrow K_{\mathcal{G}}^{0}(X, Y)$ is an isomorphism and hence it is defines an equivalence of functors.

Proof. This follows from Lemmas 3.7 and 3.6.

Lemma 3.9. The class of $\left(E^{1}, E^{0}, \alpha\right)$ in $L_{\mathcal{G}}^{1}(X, Y)$ depends only on the homotopy class of the isomorphism $\alpha$.

Proof. Let $Z=X \times[0,1], W=Y \times[0,1]$. Denote by $p: Z \rightarrow X$ the natural projection and assume that $\alpha_{t}$ is a homotopy, where $\alpha_{0}=\alpha$. Then $\alpha_{t}$ gives rise an isomorphism $\beta:\left.\left.p^{*}\left(E^{1}\right)\right|_{W} \cong p^{*}\left(E^{0}\right)\right|_{W}$, and hence to an element $\left(p^{*}\left(E^{1}\right), p^{*}\left(E^{0}\right), \beta\right)$ of $L_{\mathcal{G}}^{1}(Z, W)$. If

$$
i_{t}:(X, Y) \rightarrow(X \times\{t\}, Y \times\{t\}) \subset(Z, W),
$$

are the standard inclusions, then $\left(E^{1}, E^{0}, \alpha_{t}\right)=i_{t}^{*}\left(p^{*}\left(E^{1}\right), p^{*}\left(E^{0}\right), \beta\right)$. Consider the commutative diagram

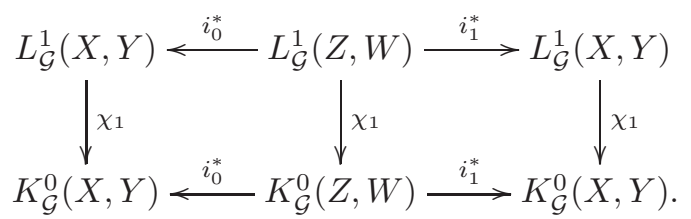

The vertical morphisms and and the morphisms of the bottom line of the above diagram are isomorphisms. Hence, the arrows of the top line are isomorphisms too. The composition $i_{0}^{*}\left(i_{1}^{*}\right)^{-1}$ is identity for the bottom line, hence it is the identity for the top line too.

The following theorem reduces the study of the functors $L_{\mathcal{G}}^{n}, n>1$, to the study of $L_{\mathcal{G}}^{1}$.

Theorem 3.10. The natural map $j_{n}: L_{\mathcal{G}}^{n}(X, Y) \rightarrow L_{\mathcal{G}}^{n+1}(X, Y)$ is an isomorphism. 
Proof. Let $E=\left(E^{0}, E^{1}, \ldots, E^{n+1} ; d_{k}\right), d_{k}:\left.\left.E^{k}\right|_{Y} \rightarrow E^{k+1}\right|_{Y}$ represent an element of the semigroup $L_{\mathcal{G}}^{n+1}(X, Y)$. To prove the surjectivity of $j_{n}$, let us first notice that $E$ is equivalent to the complex

$$
\begin{aligned}
\left(E^{0}, \ldots, E^{n-2}, E^{n-1} \oplus E^{n+1}, E^{n} \oplus E^{n+1}, E^{n+1} ;\right. \\
\left.d_{0}, \ldots, d_{n-2} \oplus 0, d_{n-1} \oplus \mathrm{Id}, d_{n} \oplus 0\right) .
\end{aligned}
$$

The maps $d_{n} \oplus 0:\left.\left.\left(E^{n} \oplus E^{n+1}\right)\right|_{Y} \rightarrow E^{n+1}\right|_{Y}$ and $0 \oplus \operatorname{Id}:\left.\left.\left(E^{n} \oplus E^{n+1}\right)\right|_{Y} \rightarrow E^{n+1}\right|_{Y}$ are homotopic within the set of surjective, $\mathcal{G}$-equivariant vector bundle morphisms $\left.\left.\left(E^{n} \oplus E^{n+1}\right)\right|_{Y} \rightarrow E^{n+1}\right|_{Y}$. Hence, by Lemma $3.2, d_{n} \oplus 0$ can be extended to a surjective morphism $b: E^{n} \oplus E^{n+1} \rightarrow E^{n+1}$ of $\mathcal{G}$-equivariant vector bundles (over the whole of $X$ ). So, the bundle $E^{n} \oplus E^{n+1}$ is isomorphic to $\operatorname{ker}(b) \oplus E^{n+1}$. Hence, the $E$ is equivalent to

$$
\left(E^{0}, \ldots, E^{n-2}, E^{n-1} \oplus E^{n+1}, \operatorname{ker}(b), 0 ; d_{0}, \ldots, d_{n-2} \oplus 0, d_{n-1}, 0\right) .
$$

This proves the surjectivity of $j_{n}$.

To prove the injectivity of $j_{n}$, it is enough to define, for any $n$, a left inverse $q_{n}: L_{\mathcal{G}}^{n}(X, Y) \rightarrow L_{\mathcal{G}}^{1}(X, Y)$ to $s_{n}:=j_{n-1} \circ \cdots \circ j_{1}$. Suppose that $\left(E^{*} ; d\right)$ represents an element of semigroup $L_{\mathcal{G}}^{n}(X, Y)$. Choose $\mathcal{G}$-invariant Hermitian metrics on $E^{i}$ and let $d_{i}^{*}:\left.\left.E^{i+1}\right|_{Y} \rightarrow E^{i}\right|_{Y}$ be the adjoint of $d_{i}$. Let

$$
F^{0}:=\bigoplus_{i} E^{2 i}, \quad F^{1}:=\bigoplus_{i} E^{2 i+1}, \quad b:\left.\left.F^{0}\right|_{Y} \rightarrow F^{1}\right|_{Y}, \quad b=\sum_{i}\left(d_{2 i}+d_{2 i+1}^{*}\right) .
$$

A standard verification shows that $b$ is an isomorphism. Since all invariant metrics are homotopic to each other, Lemma 3.9 shows that $(E, d) \rightarrow(F, b)$ defines a morphism $q_{n}: L_{\mathcal{G}}^{n}(X, Y) \rightarrow L_{\mathcal{G}}^{1}(X, Y)$. This is the desired left inverse for $s_{n}$.

Let us observe that the proof of the above theorem and Lemma 3.9 give the following corollary.

Corollary 3.11. The class of $E=\left(E^{i}, d_{i}\right)$ in $L_{\mathcal{G}}^{n}(X, Y)$ does not change if we deform the differentials $d_{i}$ continuously.

We are now ready to prove the following basic result.

Theorem 3.12. For each $n$ there exists a unique Euler characteristic

$$
\chi_{n}: L_{\mathcal{G}}^{n}(X, Y) \cong K_{\mathcal{G}}^{0}(X, Y) .
$$

In particular, $L_{\mathcal{G}}^{\infty}(X, Y) \cong K_{\mathcal{G}}^{0}(X, Y)$ and $L_{\mathcal{G}}^{n}(X, Y)$ has a natural group structure for any closed, $\mathcal{G}$-invariant subbundle $Y \subset X$.

Proof. The statement is obtained from the lemmas we have proved above as follows. First of all, Theorem 3.10 allows us to define

$$
\chi_{n}:=\chi_{1} \circ j_{1}^{-1} \circ \ldots j_{n-1}^{-1}: L_{\mathcal{G}}^{n}(X, Y) \rightarrow K_{\mathcal{G}}^{0}(X, Y) .
$$

Lemma 3.7 shows that $\chi_{n}$ is an isomorphism. The uniqueness of $\chi_{n}$ is proved in the same way as the uniqueness of $\chi_{1}$ (Lemma 3.6). 
3.3. Globally defined complexes. The above theorem provides us with an alternative definition of the groups $K_{\mathcal{G}}^{0}(X, Y)$. We now derive yet another definition of these groups that is closer to what is needed in applications and is based on differentials defined on $X$, not just on $Y$.

Let $(E, d)$ be a complex of $\mathcal{G}$-equivariant vector bundles over a $\mathcal{G}$-space $X$. A point $x \in X$ will be called a point of acyclicity of $(E, d)$ if the restriction of $(E, d)$ to $x$, i.e., the sequence of linear spaces

$$
(E, d)_{x}=\left(\ldots \stackrel{\left(d_{i}\right)_{x}}{\longrightarrow} E_{x}^{i} \stackrel{\left(d_{i+1}\right)_{x}}{\longrightarrow} E_{x}^{i+1} \stackrel{\left(d_{i+2}\right)_{x}}{\longrightarrow} \cdots\right),
$$

is exact. The support $\operatorname{supp}(E, d)$ of the finite complex $(E, d)$ is the complement in $X$ of the set of its points of acyclicity. This definition and the following lemma hold also for $X$ non-compact.

Lemma 3.13. The support $\operatorname{supp}(E, d)$ is a closed $\mathcal{G}$-invariant subspace of $X$.

Proof. The fact that $\operatorname{supp}(E, d)$ is closed is classical (see $[2,10]$ for example). The invariance should be checked up over one fiber of $X$ at $b \in B$. But this is once again a well known fact of equivariant $K$-theory (see e.g. [10]).

Lemma 3.14. Let $E^{n}, \ldots, E^{0}$ be $\mathcal{G}$-equivariant vector bundles over $X$ and $Y$ be a closed, $\mathcal{G}$-invariant subbundle of $X$. Suppose there are given morphisms $d_{i}$ : $\left.\left.E^{i}\right|_{Y} \rightarrow E^{i-1}\right|_{Y}$ such that $\left(\left.E^{i}\right|_{Y}, d_{i}\right)$ is an exact complex. Then the morphisms $d_{i}$ can be extended to morphisms defined over $X$ such that we still have a complex of $\mathcal{G}$-equivariant vector bundles.

Proof. We will show that we can extend each $d_{i}$ to a morphism $r_{i}: E^{i} \rightarrow E^{i-1}$ such that $r_{i-1} \circ r_{i}=0$. Let us find a $\mathcal{G}$-invariant open neighborhood $U$ of $Y$ in $X$ such that for any $i$ there exist an extension $s_{i}$ of $d_{i}$ to $U$ with $(E, s)$ still an exact sequence. The desired $r_{i}$ will be then be defined as $r_{i}=\rho s_{i}$, where $\rho: X \rightarrow[0,1]$ is a continuous function, $\rho=1$ on $Y$ and $\operatorname{supp} \rho \subset U$.

Let us construct $U$ by induction over $i$. Assume that for the closure $\bar{U}_{i}$ of some open $\mathcal{G}$-neighborhood of $Y$ in $X$ we can extend $d_{j}$ to $s_{j}(j=1, \ldots, i)$ such that on $\bar{U}_{i}$ the sequence

$$
E^{i} \stackrel{s_{i}}{\longrightarrow} E^{i-1} \stackrel{s_{i-1}}{\longrightarrow} \cdots \rightarrow E^{0} \rightarrow 0
$$

is exact. Suppose, $K_{i}:=\operatorname{ker}\left(s_{i} \mid \bar{U}_{i}\right)$. Then $d_{i+1}$ determines a cross section of the bundle $\left.\operatorname{Hom}_{\mathcal{G}}\left(E^{i}, K_{i}\right)\right|_{Y}$. This section can be extended to an open $\mathcal{G}$-neighborhood $V$ of $Y$ in $\bar{U}_{i}$. We hence obtain an extension $s_{i+1}: E^{i+1} \rightarrow K_{i}$ of $d_{i+1}: E^{i+1} \rightarrow K_{i}$ over $V$. Since $\left.d_{i+1}\right|_{Y}$ is surjective (with range $K_{i}$ ), the morphism $s_{i+1}$ will be surjective $\bar{U}_{i+1}$ for some open $U_{i+1} \subset U_{i}$.

The above lemma suggests the following definition.

Definition 3.15. Let $X$ be a compact $\mathcal{G}$-bundle and $Y \subset X$ be a $\mathcal{G}$-invariant subbundle. We define $E_{\mathcal{G}}^{n}(X, Y)$ to be the semigroup of homotopy classes of complexes of $\mathcal{G}$-equivariant vector bundles of length $n$ over $X$ such that their restrictions to $Y$ are acyclic (i.e., exact).

We shall say that two complexes are homotopic if they are isomorphic to the restrictions to $X \times\{0\}$ and $X \times\{1\}$ of a complex defined over $X \times I$ and acyclic over $Y \times I$.

Remark 3.16. By Corollary 3.11, the restriction of morphisms induces a morphism $\Phi^{n}: E_{\mathcal{G}}^{n}(X, Y) \rightarrow L_{\mathcal{G}}^{n}(X, Y)$. 
Theorem 3.17. Let $X$ be a compact $\mathcal{G}$-bundle and $Y \subset X$ be a $\mathcal{G}$-invariant subbundle. Then the natural transformation $\Phi_{n}$, defined in the above remark, is an isomorphism.

Proof. The surjectivity of $\Phi_{n}$ follows from 3.14. The injectivity of $\Phi_{n}$ can be proved in the same way as [2, Lemma 2.6.13], keeping in mind Lemma 3.14.

More precisely, we need to demonstrate that differentials of any complex over $\mathcal{G}$-subbundle $(X \times\{0\}) \cup(X \times\{1\}) \cup(Y \times I)$ of $X \times I$, which is acyclic over $Y \times I$, can be extended to a complex over the entire $X \times I$. The desired construction has the following three stages. First, let $V$ be a $\mathcal{G}$-invariant neighborhood of $Y$ such that the restriction of our complex is still acyclic on $(V \times\{0\}) \cup(V \times\{1\}) \cup(Y \times I)$ as well as on its closure $(\bar{V} \times\{0\}) \cup(\bar{V} \times\{1\}) \cup(Y \times I)$. By Lemma 3.14, one can extend the differentials $d_{i}$ to $\mathcal{G}$-equivariant morphisms $r_{i}$ over $\bar{V} \times I$ that still define a complex. Second, let $\rho_{1}, \rho_{2}$ be a $\mathcal{G}$-invariant partition of unity subordinated to covering $V \times I,(X \backslash Y) \times I$ of $X \times Y$. Let us extend original differentials to $(X \times[0,1 / 4]) \cup(X \times[3 / 4,1]) \cup(V \times I)$ by taking $d_{i}(x, t):=d_{i}(x, 0)$ for $t \leq \frac{1}{4} \cdot \rho_{2}(x)$, $x \in X \backslash Y$. Similarly near $t=1$. Also,

$$
d_{i}(x, t):=r_{i}\left(x, \frac{\left(t-\frac{1}{4} \cdot \rho_{2}(x)\right)}{\left(1-\frac{1}{2} \cdot \rho_{2}(x)\right)}\right), \quad x \in V .
$$

Third, multiplying this differential by a function $\tau: X \times I \rightarrow I$ equal to 1 on the original subset of definition of the differential and to 0 off $(X \times[0,1 / 4]) \cup(X \times$ $[3 / 4,1]) \cup(V \times I)$ we obtain the desired extension.

3.4. The non-compact case. In the case of a locally compact, paracompact $\mathcal{G}^{-}$ bundle $X$, we change the definitions of $L_{\mathcal{G}}^{n}$ and $E_{\mathcal{G}}^{n}$ as follows. In the definition of $L_{\mathcal{G}}^{n}$, the morphisms $d_{i}$ have to be defined and form an exact sequence off the interior of some compact $\mathcal{G}$-invariant subset $C$ of $X \backslash Y$ (the complement of $Y$ in $X)$. In the definition of $E_{\mathcal{G}}^{n}$, the complexes have to be exact outside some compact $\mathcal{G}$-invariant subset of $X \backslash Y$. In other words, $L_{\mathcal{G}}^{n}(X, Y)=L_{\mathcal{G}}^{n}\left(X^{+}, Y^{+}\right)$.

Since the proof of Lemma 3.14 is still valid, we have the analogue of Theorem 3.17: there is a natural isomorphism

$$
L_{\mathcal{G}}^{n}(X, Y) \cong E_{\mathcal{G}}^{n}(X, Y) .
$$

The proof of the other statement also can be extended to the non-compact case. The only difference is that we have to replace $Y$ with $X \backslash U$, where $U$ is an open, $\mathcal{G}^{-}$ invariant subset with compact closure. Then, when we study two element sequences $E=\left(E^{i}, d_{i}\right)$, we have to take the unions of the corresponding open sets. Of course, these sets are not bundles, unlike $Y$, but for our argument using extensions this is not dangerous. This ultimately gives

$$
K_{\mathcal{G}}^{0}(X, Y) \cong L_{\mathcal{G}}^{n}(X, Y) \cong E_{\mathcal{G}}^{n}(X, Y), \quad n \geq 1
$$

As we shall see below, the liberty of using these equivalent definitions of $K_{\mathcal{G}}^{0}(X, Y)$ is quite convenient in applications, especially when studying products.

\section{The Thom isomorphism}

In this section, we establish the Thom isomorphism in gauge-equivariant $K$ theory. We begin with a discussion of products and of the Thom morphism. 
4.1. Products. Let $\pi_{X}: X \rightarrow B$ be a $\mathcal{G}$-space, $\tilde{\pi}_{F}: F \rightarrow X$ be a complex $\mathcal{G}-$ vector bundle over $X$, and $s: X \rightarrow F$ a $\mathcal{G}$-invariant section. We shall denote by $\Lambda^{i} F$ the $i$-th exterior power of $F$, which is again a complex $\mathcal{G}$-equivariant vector bundle over $X$. As in the proof of the Thom isomorphism for ordinary vector bundles, we define the complex $\Lambda(F, s)$ of $\mathcal{G}$-equivariant vector bundles over $X$ by

$$
\Lambda(F, s):=\left(0 \rightarrow \Lambda^{0} F \stackrel{\alpha^{0}}{\longrightarrow} \Lambda^{1} F \stackrel{\alpha^{1}}{\longrightarrow} \ldots \stackrel{\alpha^{n-1}}{\longrightarrow} \Lambda^{n} F \rightarrow 0\right),
$$

where $\alpha^{k}\left(v_{x}\right)=s(x) \wedge v_{x}$ for $v_{x} \in \Lambda^{k} F^{x}$ and $n=\operatorname{dim} F$. It is immediate to check that $\alpha^{j+1}(x) \alpha^{j}(x)=0$, and hence that $(\Lambda(F, s), \alpha)$ is indeed a complex.

The Künneth formula shows that the complex $\Lambda(F, s)$ is acyclic for $s(x) \neq 0$, and hence $\operatorname{supp}(\Lambda(F, s)):=\{x \in X \mid s(x)=0\}$. If this set is compact, then the results of Section 3 will associate to the complex $\Lambda(F, s)$ of Equation (10) an element

$$
[\Lambda(F, s)] \in K_{\mathcal{G}}^{0}(X) .
$$

Let $X$ be a $\mathcal{G}$-bundle and $\pi_{F}: F \rightarrow X$ be a $\mathcal{G}$-equivariant vector bundle over $X$. The point of the above construction is that $\pi_{F}^{*}(F)$, the lift of $F$ back to itself, has a canonical section whose support is $X$. Let us recall how this is defined. Let $\pi_{F F}: \pi_{F}^{*}(F) \rightarrow F$ be the $\mathcal{G}$-vector bundle over $F$ with total space

$$
\pi_{F}^{*}(F):=\left\{\left(f_{1}, f_{2}\right) \in F \times F, \pi_{F}\left(f_{1}\right)=\pi_{F}\left(f_{2}\right)\right\}
$$

and $\pi_{F F}\left(f_{1}, f_{2}\right)=f_{1}$. The vector bundle $\pi_{F F}: \pi_{F}^{*}(F) \rightarrow F$ has the canonical section

$$
s_{F}: F \rightarrow \pi_{F}^{*} F, \quad s_{F}(f)=(f, f) .
$$

The support of $s_{F}$ is equal to $X$. Hence, if $X$ is a compact space, using again the results of Section 3, especially 3.17, we obtain an element

$$
\lambda_{F}:=\left[\Lambda\left(\pi_{F}^{*}(F), s_{F}\right)\right] \in K_{\mathcal{G}}^{0}(F) .
$$

Recall that the tensor product of vector bundles defines a natural product $a b=$ $a \otimes b \in K_{\mathcal{G}}^{0}(X)$ for any $a \in K_{\mathcal{G}}^{0}(B)$ and any $b \in K_{\mathcal{G}}^{0}(X)$, where $\pi_{X}: X \rightarrow B$ is a compact $\mathcal{G}$-space, as above.

Recall that all our vector bundles are assumed to be complex vector bundles, except for the ones coming from geometry (tangent bundles, their exterior powers) and where explicitly mentioned. Due to the importance that $F$ be complex in the following definition, we shall occasionally repeat this assumption.

Definition 4.1. Let $\pi_{F}: F \rightarrow X$ be a (complex) $\mathcal{G}$-equivariant vector bundle. Assume the $\mathcal{G}$-bundle $X \rightarrow B$ is compact and let $\lambda_{F} \in K_{\mathcal{G}}^{0}(F)$ be the class defined in Equation (12), then the mapping

$$
\varphi^{F}: K_{\mathcal{G}}^{0}(X) \rightarrow K_{\mathcal{G}}^{0}(F), \quad \varphi^{F}(a)=\pi_{F}^{*}(a) \otimes \lambda_{F} .
$$

is called the Thom morphism.

As we shall see below, the definition of the Thom homomorphism extends to the case when $X$ is not compact, although the Thom element itself is not defined if $X$ is not compact.

The definition of the Thom isomorphism immediately gives the following proposition. We shall use the notation of Proposition 4.1.

Proposition 4.2. The Thom morphism $\varphi^{F}: K_{\mathcal{G}}^{0}(X) \rightarrow K_{\mathcal{G}}^{0}(F)$ is a morphism of $K_{\mathcal{G}}^{0}(B)$-modules. 
Let $\iota: X \hookrightarrow F$ be the zero section embedding of $X$ into $F$. Then $\iota$ induces homomorphisms

$$
\iota^{*}: K_{\mathcal{G}}^{0}(F) \rightarrow K_{\mathcal{G}}^{0}(X) \text { and } \iota^{*} \circ \varphi^{F}: K_{\mathcal{G}}^{0}(X) \rightarrow K_{\mathcal{G}}^{0}(X) .
$$

It follows from the definition that $\iota^{*} \varphi^{F}(a)=a \cdot \sum_{i=0}^{n}(-1)^{i} \Lambda^{i} F$.

4.2. The non-compact case. We now consider now the case when $X$ is locally compact, but not necessarily compact. The complex $\Lambda\left(\pi_{F}^{*}(F), s_{F}\right)$ has a noncompact support, and hence it does not define an element of $K_{\mathcal{G}}^{0}(F)$. However, if $a=[(E, \alpha)] \in K_{\mathcal{G}}^{0}(X)$ is represented by the complex $(E, \alpha)$ of vector bundles with compact support (Section 3), then we can still consider the tensor product complex

$$
\left(\pi_{F}^{*}(\mathcal{E}), \pi_{F}^{*}(\alpha)\right) \otimes \Lambda\left(\pi_{F}^{*} F, s_{F}\right)
$$

From the Künneth formula for the homology of a tensor product we obtain that the support of a tensor product complex is the intersection of the supports of the two complexes. In particular, we obtain

$$
\begin{aligned}
\operatorname{supp}\left\{\left(\pi_{F}^{*} E, \pi_{F}^{*} \alpha\right) \otimes \Lambda\left(\pi_{F}^{*} F, s_{F}\right)\right\} \subset & \operatorname{supp}\left(\pi_{F}^{*} E, \pi_{F}^{*} \alpha\right) \cap \operatorname{supp} \Lambda\left(\pi_{F}^{*} F, s_{F}\right) \subset \\
& \subset \operatorname{supp}\left(\pi_{F}^{*} E, \pi_{F}^{*} \alpha\right) \cap X=\operatorname{supp}(E, \alpha) .
\end{aligned}
$$

Thus, the complex $\left(\pi_{F}^{*} \mathcal{E}, \pi_{E}^{*} \alpha\right) \otimes \Lambda\left(\pi_{F}^{*} F, s_{F}\right)$ has compact support and hence defines an element in $K_{\mathcal{G}}^{0}(F)$.

Proposition 4.3. The homomorphism of $K_{\mathcal{G}}^{0}(B)$-modules

$$
\varphi^{F}: K_{\mathcal{G}}^{0}(X) \rightarrow K_{\mathcal{G}}^{0}(F), \quad \varphi^{F}(a)=\left[\left(\pi_{F}^{*} \mathcal{E}, \pi_{F}^{*} \alpha\right) \otimes \Lambda\left(\pi_{F}^{*} F, s_{F}\right)\right],
$$

defined in Equation (13) extends the Thom morphism to the case of not necessarily compact $X$. The Thom morphism $\varphi^{F}$ satisfies

$$
i^{*} \varphi^{F}(a)=a \cdot \sum_{i=0}^{n}(-1)^{i} \Lambda^{i} F
$$

in the non-compact case as well.

Let $F \rightarrow X$ be a $\mathcal{G}$-equivariant vector bundle and $F^{1}=F \times \mathbb{R}$, regarded as a vector bundle over $X \times \mathbb{R}$. The periodicity isomorphisms in gauge-equivariant $K$-theory groups [15, Theorem 3.18]

$$
K_{\mathcal{G}}^{i \pm 1}(X \times \mathbb{R}, Y \times \mathbb{R}) \simeq K_{\mathcal{G}}^{i}(X, Y)
$$

can be composed with $\varphi^{F^{1}}$, the Thom morphism for $F^{1}$, giving a morphism

$$
\varphi^{F}: K_{\mathcal{G}}^{i}(X) \rightarrow K_{\mathcal{G}}^{i}(F), \quad i=0,1 .
$$

This morphism is the Thom morphism for $K^{1}$.

Let $p_{X}: X \rightarrow B$ and $p_{Y}: Y \rightarrow B$ be two compact $\mathcal{G}$-fiber bundles. Let $\pi_{E}: E \rightarrow X$ and $\pi_{F}: F \rightarrow Y$ be two complex $\mathcal{G}$-equivariant vector bundles. Denote by $p_{1}: X \times_{B} Y \rightarrow X$ and by $p_{2}: X \times_{B} Y \rightarrow Y$ the projections onto the two factors and define $E \otimes F:=p_{1}^{*} E \otimes p_{2}^{*} F$. The $\mathcal{G}$-equivariant vector bundle $E \otimes F$ will be called the external tensor product of $E$ and $F$ over $B$. It is a vector bundle over $X \times_{B} Y$. Then the formula

$$
K_{\mathcal{G}}^{0}(X) \otimes K_{\mathcal{G}}^{0}(Y) \ni[E] \otimes[F] \rightarrow[E] \otimes[F]:=[E \otimes F] \in K_{\mathcal{G}}^{0}\left(X \times_{B} Y\right)
$$

defines a product $K_{\mathcal{G}}^{0}(X) \otimes K_{\mathcal{G}}^{0}(Y) \rightarrow K_{\mathcal{G}}^{0}\left(X \times{ }_{B} Y\right)$. 
In particular, consider two complex $\mathcal{G}$-equivariant vector bundles $\pi_{E}: E \rightarrow X$ and $\pi_{F}: F \rightarrow X$. Then $E \oplus F=E \times_{X} F$ and we obtain a product

$$
K_{\mathcal{G}}^{i}(E) \otimes K_{\mathcal{G}}^{j}(F) \ni[E] \otimes[F] \rightarrow[E] \otimes[F]:=[E \otimes F] \rightarrow K_{\mathcal{G}}^{i+j}(E \oplus F) .
$$

Using also periodicity, we obtain the product

$$
\otimes: K_{\mathcal{G}}^{i}(E) \otimes K_{\mathcal{G}}^{j}(F) \rightarrow K_{\mathcal{G}}^{i+j}(E \oplus F) .
$$

This product is again seen to be given by the tensor product of the (lifted) complexes (when representing $K$-theory classes by complexes) as in the classical case.

The external product $\otimes$ behaves well with respect to the "Thom construction," in the following sense. Let $F^{1}$ and $F^{2}$ be two complex bundles over $X$, and $s_{1}, s_{2}$ two corresponding sections of these bundles. Then

$$
\Lambda\left(F^{1} \oplus F^{2}, s_{1} \otimes 1+1 \otimes s_{2}\right)=\Lambda\left(F^{1}, s_{1}\right) \otimes \Lambda\left(F^{2}, s_{2}\right) .
$$

In particular, if $X$ is compact, we obtain

$$
\lambda_{E} \bigotimes \lambda_{F}=\lambda_{E \oplus F} .
$$

We shall write $s_{1}+s_{2}=s_{1} \otimes 1+1 \otimes s_{2}$, for simplicity.

The following theorem states that the Thom class is multiplicative with respect to direct sums of vector bundles (see also [5]).

Theorem 4.4. Let $E, F \rightarrow X$ be two $\mathcal{G}$-equivariant vector bundles, and regard $E \oplus F \rightarrow E$ as the $\mathcal{G}$-equivariant vector bundle $\pi_{E}^{*}(F)$ over $E$. Then $\varphi^{\pi_{E}^{*}(F)} \circ \varphi^{E}=$ $\varphi^{E \oplus F}$.

The above theorem amounts to the commutativity of the diagram

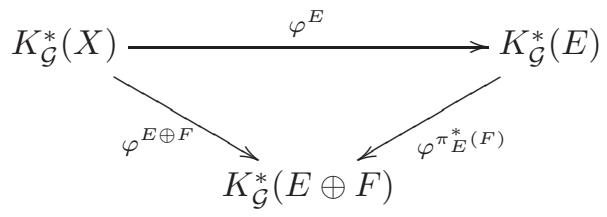

Proof. Let $F^{1}:=\pi_{E}^{*}(F)=E \oplus F$, regarded as a vector bundle over $E$. Consider the projections

$$
\pi_{E}: E \rightarrow X, \quad \pi_{F}: F \rightarrow X, \quad \pi_{E \oplus F}: E \oplus F \rightarrow X, \quad t=\pi_{F^{1}}: E \oplus F \rightarrow E .
$$

Let $x \in K_{\mathcal{G}}^{0}(X)$. Then $\varphi^{E}(x)=\pi_{E}^{*}(x) \otimes \Lambda\left(\pi_{E}^{*}(E), s_{E}\right)$. Now we use that $t^{*} \pi_{E}^{*}(x)=$ $\pi_{E \oplus F}^{*}(x)$ and $t^{*} \Lambda\left(\pi_{E}^{*}(E), s_{E}\right)=\Lambda\left(\pi_{E \oplus F}^{*}(E), s_{E} \circ t\right)$. Since $s_{E} \circ t+s_{F^{1}}=s_{E \oplus F}$, Equations (18) and (19) then give

$$
\Lambda\left(\pi_{E \oplus F}^{*}(E \oplus F), s_{E \oplus F}\right)=\Lambda\left(\pi_{E \oplus F}^{*}(E), s_{E} \circ t\right) \otimes \Lambda\left(\pi_{E \oplus F}^{*}(F), s_{F^{1}}\right) .
$$

Putting together the above calculations we obtain

$$
\begin{aligned}
& \varphi^{F^{1}} \varphi^{E}(x)=t^{*}\left(\varphi^{E}(x)\right) \otimes \Lambda \\
&\left.=t^{*} \pi_{E}^{*}(x) \otimes t^{*}\left(F^{1}\right), s_{F^{1}}\right) \\
&\left.=\pi_{E \oplus F}^{*}(x) \otimes \Lambda\left(\pi_{E}^{*}(E), s_{E}\right)\right) \otimes \Lambda\left(t^{*}\left(F^{1}\right), s_{F^{1}}\right) \\
& \quad=\pi_{E \oplus F}^{*}(x) \otimes \Lambda\left(s_{E} \circ t\right) \otimes \Lambda\left(\pi_{E \oplus F}^{*} F, s_{F^{1}}\right) \\
&
\end{aligned}
$$

The proof is now complete. 
We are now ready to formulate and prove the Thom isomorphism in the setting of gauge-equivariant vector bundles.

Theorem 4.5. Let $X \rightarrow B$ be a $\mathcal{G}$-bundle and $F \rightarrow X$ a complex $\mathcal{G}$-equivariant vector bundle, then $\varphi^{F}: K_{\mathcal{G}}^{i}(X) \rightarrow K_{\mathcal{G}}^{i}(F)$ is an isomorphism.

Proof. Assume first that $F$ is a trivial bundle, that is, that $F=X \times_{B} \mathcal{V}$, where $\mathcal{V} \rightarrow B$ is a complex, finite-dimensional $\mathcal{G}$-equivariant vector bundle. We continue to assume that $B$ is compact.

Let us denote by $\mathbb{C}:=B \times \mathbb{C}$ the 1 -dimensional $\mathcal{G}$-bundle with the trivial action of $\mathcal{G}$ on $\mathbb{C}$. Also, let us denote by $P(\mathcal{V} \oplus \underline{\mathbb{C}})$ the projective space associated to $\mathcal{V} \oplus \mathbb{C}$. As a topological space, $P(\mathcal{V} \oplus \mathbb{C})$ identifies with the fiberwise one-point compactification of $\mathcal{V}$. The embeddings $\mathcal{V} \subset P(\mathcal{V} \oplus \mathbb{C})$ and $\mathcal{V} \times_{B} X \subset P(\mathcal{V} \oplus \underline{\mathbb{C}}) \times_{B} X$ then gives rise to the following natural morphism (Equation (6))

$$
j: K_{\mathcal{G}}^{0}(\mathcal{V}) \rightarrow K_{\mathcal{G}}^{0}(P(\mathcal{V} \oplus \underline{\mathbb{C}})), \quad j: K_{\mathcal{G}}^{0}\left(\mathcal{V} \times_{B} X\right) \rightarrow K_{\mathcal{G}}^{0}\left(P(\mathcal{V} \oplus \underline{\mathbb{C}}) \times_{B} X\right) .
$$

Let $X$ be compact and let $x \in K_{\mathcal{G}}^{0}\left(P(\mathcal{V} \oplus \underline{\mathbb{C}}) \times_{B} X\right)$ be arbitrary. The fibers of the projectivization $P(V \oplus \underline{\mathbb{C}})$ are complex manifolds, so we can consider the analytical index of the correspondent family of Dolbeault operators over $P(V \oplus 1)$ with coefficients in $x$ (cf. [3, page 123]). This index is an element of $K_{\mathcal{G}}^{0}(X)$ by the results of [15]. Taking the composition with $j$ (cf. [3, page 122-123]) we get a family of mappings $\alpha_{X}: K_{\mathcal{G}}^{0}\left(\mathcal{V} \times_{B} X\right) \rightarrow K_{\mathcal{G}}^{0}(X)$, having the following properties:

(i) $\alpha_{X}$ is functorial with the respect to $\mathcal{G}$-equivariant morphisms;

(ii) $\alpha_{X}$ is a morphism of $K_{\mathcal{G}}^{0}(X)$-modules;

(iii) $\alpha_{B}\left(\lambda_{V}\right)=1 \in K_{\mathcal{G}}^{0}(B)$.

Let $X^{+}:=X \cup B$ be the fiberwise one-point compactification of $X$. The commutative diagram

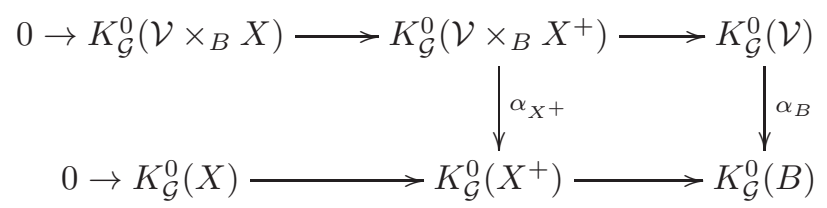

with exact lines allows us to define $\alpha_{X}$ for $X$ non-compact.

Let $x \in K_{\mathcal{G}}^{0}(X)$, then by (ii)

$$
\alpha_{X}\left(\lambda_{V} x\right)=\alpha_{X}\left(\lambda_{\mathcal{V}}\right) x=x, \quad \alpha \varphi=\mathrm{Id} .
$$

Let $q:=\pi_{F}: F=\mathcal{V} \times_{B} X \rightarrow X, p: X \times_{B} \mathcal{V} \rightarrow X, q_{1}: \mathcal{V} \times_{B} X \times_{B} \mathcal{V} \rightarrow \mathcal{V}$ (onto the first entry), $q_{2}: X \times_{B} \mathcal{V} \rightarrow B$, and by $\widetilde{y} \in K_{\mathcal{G}}^{0}\left(X \times_{B} \mathcal{V}\right)$ we denote the element, obtained from $y$ under the mapping $X \times_{B} \mathcal{V} \rightarrow \mathcal{V} \times_{B} X,(x, v) \mapsto(-v, x)$ (such that $\mathcal{V} \times_{B} X \times_{B} \mathcal{V} \rightarrow \mathcal{V} \times_{B} X \times_{B} \mathcal{V},(u, x, v) \mapsto(-v, x, u)$ is homotopic to the identity).

Let $y \in K_{\mathcal{G}}^{0}\left(\mathcal{V} \times_{B} X\right)$, then once again by (i), (ii) and then by (iii)

$$
\begin{gathered}
\varphi\left(\alpha_{X}(y)\right)=\pi_{E}^{*} \alpha_{X}(y) \otimes q^{*} \lambda_{\mathcal{V}}=\alpha_{X \times_{B} \mathcal{V}}\left(p_{1}^{*} y\right) \otimes q^{*} \lambda_{\mathcal{V}}=\alpha_{X \times_{B} \mathcal{V}}\left(p_{1}^{*} y \otimes q^{*} \lambda_{V}\right) \\
=\alpha_{X \times_{B}} \mathcal{V}\left(y \otimes \lambda_{V}\right)=\alpha_{X \times_{B} \mathcal{V}}\left(\lambda_{V} \otimes \widetilde{y}\right)=\alpha_{X \times_{B} \mathcal{V}}\left(q_{1}^{*} \lambda_{V} \otimes \widetilde{y}\right) \\
=\alpha_{X \times_{B} \mathcal{V}}\left(q_{1}^{*} \lambda_{V}\right) \otimes \widetilde{y}=q_{2}^{*} \alpha_{B}\left(\lambda_{V}\right) \otimes \widetilde{y}=q_{2}^{*}(1) \otimes \widetilde{y}=\widetilde{y} \in K_{\mathcal{G}}^{0}\left(X \times_{B} \mathcal{V}\right),
\end{gathered}
$$

We obtain that $\varphi \circ \alpha_{X}$ is an isomorphism. Since $\alpha_{X} \circ \varphi=\mathrm{Id}, \alpha_{X}$ is the two-sides inverse of $\varphi$ and the automorphism $\varphi \circ \alpha_{X}$ is the identity. 
The proof for a general (complex) $\mathcal{G}$-equivariant vector bundle $F \rightarrow X$ can be done as in [3, p. 124]. However, we found it more convenient to use the following argument. Embed first $F$ into a trivial bundle $E=\mathcal{V} \times_{B} X$. Let $\varphi^{1}$ and $\varphi^{2}$ be the Thom maps associated to the bundles $E \rightarrow F$ and $E \rightarrow X$. Then by Theorem 4.4 the diagram

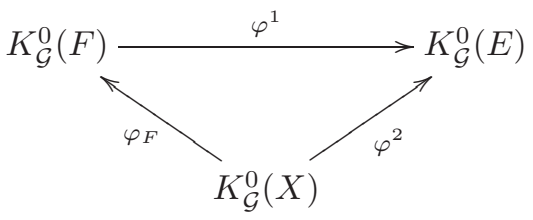

is commutative, while $\varphi^{2}$ is an isomorphism by the first part of the proof. Therefore $\varphi_{F}$ is injective. The same argument show that $\varphi^{1}$ is injective. But $\varphi^{1}$ must also be surjective, because $\varphi^{2}$ is an isomorphism. Thus, $\varphi^{1}$ is an isomorphism, and hence $\varphi^{2}$ is an isomorphism too.

\section{GYSIN MAPS}

We now discuss a few constructions related to the Thom isomorphism, which will be necessary for the definition of the topological index. The most important one is the Gysin map. For several of the constructions below, the setting of $\mathcal{G}$-spaces and even $\mathcal{G}$-bundles is too general, and we shall have to consider longitudinally smooth $\mathcal{G}$-fiber bundles $\pi_{X}: X \rightarrow B$. The main reason why we need longitudinally smooth bundles to define the Gysin map is the same as in the definition of the Gysin map for embeddings of smooth manifolds. We shall denote by $T_{\text {vert }} X$ the vertical tangent bundle to the fibers of $X \rightarrow B$. All tangent bundles below will be vertical tangent bundles.

Let $X$ and $Y$ be longitudinally smooth $\mathcal{G}$-fiber bundles, $i: X \rightarrow Y$ be an equivariant fiberwise embedding, and $p_{T}: T_{\text {vert }} X \rightarrow X$ be the vertical tangent bundle to $X$. Assume $Y$ is equipped with a $\mathcal{G}$-invariant Riemannian metric and let $p_{N}: N_{\text {vert }} \rightarrow X$ be the fiberwise normal bundle to the image of $i$

Let us choose a function $\varepsilon: X \rightarrow(0, \infty)$ such that the map of $N_{\text {vert }}$ to itself

$$
n \mapsto \varepsilon \frac{n}{1+|n|}
$$

is $\mathcal{G}$-equivariant and defines a $\mathcal{G}$-diffeomorphism $\Phi: N_{\text {vert }} \rightarrow W$ onto a bundle of open tubular neighborhoods $W \supset X$ in $Y$.

Let $(N \oplus N)_{\text {vert }}:=N_{\text {vert }} \oplus N_{\text {vert }}$. The embedding $i: X \rightarrow Y$ can be written as a composition of two fiberwise embeddings $i_{1}: X \rightarrow W$ and $i_{2}: W \rightarrow Y$. Passing to differentials we obtain

$$
T_{\text {vert }} X \stackrel{d i_{1}}{\longrightarrow} T_{\text {vert }} W \stackrel{d i_{2}}{\longrightarrow} T_{\text {vert }} Y \quad \text { and } \quad d \Phi: T_{\text {vert }} N \rightarrow T_{\text {vert }} W,
$$

where we use the simplified notation $T_{\text {vert }} N=T_{\text {vert }} N_{\text {vert }}$.

Lemma 5.1. (cf. [10, page 112]) The manifold $T_{\text {vert }} N$ can be identified with $p_{T}^{*}(N \oplus N)_{\text {vert }}$ with the help of a $\mathcal{G}$-equivariant diffeomorphism $\psi$ such that makes 
the following diagram commutative

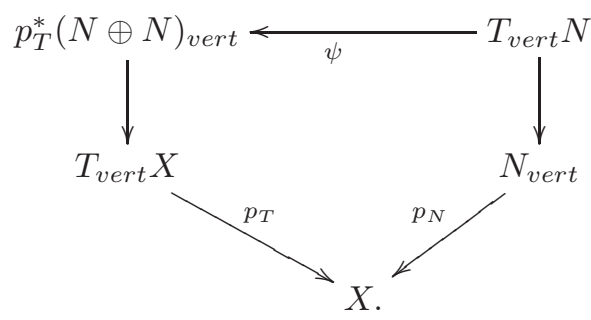

Proof. The vertical tangent bundle $T_{\text {vert }} N \rightarrow N_{\text {vert }}$ and the vector bundle

$$
p_{N}^{*}\left(T_{\text {vert }} X\right) \oplus p_{N}^{*}\left(N_{\text {vert }}\right) \rightarrow N_{\text {vert }}
$$

are isomorphic as $\mathcal{G}$-equivariant vector bundles over $N_{\text {vert }}$.

Indeed, a point of the total space $T_{\text {vert }} N$ is a pair of the form $\left(n_{1}, t+n_{2}\right)$, where both vectors are from the fiber over the point $x \in X$. Similarly, we represent elements $p_{T}^{*}(N \oplus N)_{\text {vert }}$ as pairs of the form $\left(t, n_{1}+n_{2}\right)$. Let us define $\psi$ by the equality $\psi\left(n_{1}, t+n_{2}\right)=\left(t, n_{1}+n_{2}\right)$.

With the help of the relation $i \cdot\left(n_{1}, n_{2}\right)=\left(-n_{2}, n_{1}\right)$, we can equip

$$
p_{T}^{*}(N \oplus N)_{\text {vert }}=p_{T}^{*}\left(N_{\text {vert }}\right) \oplus p_{T}^{*}\left(N_{\text {vert }}\right)
$$

with a structure of a complex manifold. Then we can consider the Thom homomorphism

$$
\varphi: K_{\mathcal{G}}^{0}\left(T_{\text {vert }} X\right) \rightarrow K_{\mathcal{G}}^{0}\left(p_{T}^{*}(N \oplus N)_{\text {vert }}\right) .
$$

Since $T_{\text {vert }} W$ is an open $\mathcal{G}$-stable subset of $T_{\text {vert }} Y$ and $d i_{2}: T_{\text {vert }} W \rightarrow T_{\text {vert }} Y$ is a fiberwise embedding, by Equation 6, there is the homomorphism $\left(d i_{2}\right)_{*}$ : $K_{\mathcal{G}}^{0}\left(T_{\text {vert }} W\right) \rightarrow K_{\mathcal{G}}^{0}\left(T_{\text {vert }} Y\right)$.

Definition 5.2. Let $i: X \rightarrow Y$ be an equivariant embedding of $\mathcal{G}$-bundles. The Gysin homomorphism is the mapping

$$
i_{!}: K_{\mathcal{G}}^{0}\left(T_{\text {vert }} X\right) \rightarrow K_{\mathcal{G}}^{0}\left(T_{\text {vert }} Y\right), \quad i_{!}=\left(d i_{2}\right)_{*} \circ\left(d \Phi^{-1}\right)^{*} \circ \psi^{*} \circ \varphi .
$$

In other words, it is obtained by passage to $K$-groups in the upper part of the diagram

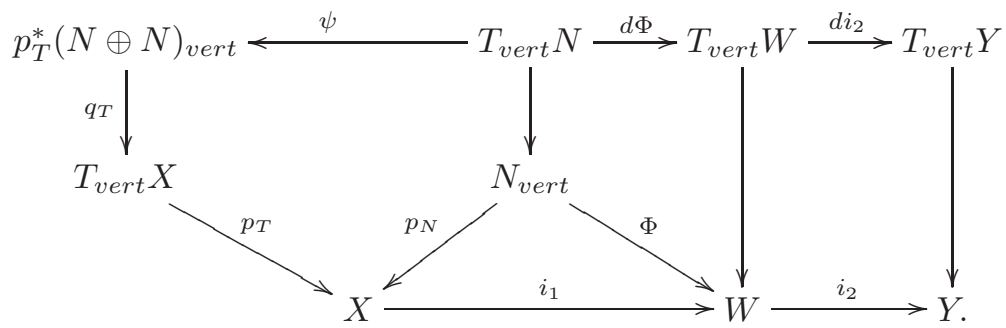

Another choice of metric and neighborhood $W$ induces the homotopic map and (by the item 3 of Theorem 5.3 below) the same homomorphism.

Theorem 5.3 (Properties of Gysin homomorphism). Let $i: X \rightarrow Y$ be a $\mathcal{G}$-embedding.

(i) $i_{\text {! }}$ is a homomorphism of $K_{\mathcal{G}}^{0}(B)$-modules.

(ii) Let $i: X \rightarrow Y$ and $j: Y \rightarrow Z$ be two fiberwise $\mathcal{G}$-embeddings, then $(j \circ i)_{\text {! }}=$ $j ! \circ i_{\text {! }}$ 
(iii) Let fiberwise embeddings $i_{1}: X \rightarrow Y$ and $i_{2}: X \rightarrow Y$ be $\mathcal{G}$-homotopic in the class of embeddings. Then $\left(i_{1}\right) !=\left(i_{2}\right) !$.

(iv) Let $i_{!}: X \rightarrow Y$ be a fiberwise $\mathcal{G}$-diffeomorphism, then $i_{!}=\left(d i^{-1}\right)^{*}$.

(v) A fiberwise embedding $i: X \rightarrow Y$ can be represented as a compositions of embeddings $X$ in $N_{\text {vert }}$ (as the zero section $s_{0}: x \rightarrow N$ ) and $N_{\text {vert }} \rightarrow Y$ by $i_{2} \circ \Phi: N_{\text {vert }} \rightarrow Y$. Then $i_{!}=\left(i_{2} \circ \Phi\right) !\left(s_{0}\right) !$.

(vi) Consider the complex bundle $p_{T}^{*}\left(N_{\text {vert }} \otimes \mathbb{C}\right)$ over $T_{\text {vert }} X$. Let us form the complex $\Lambda\left(p_{T}^{*}\left(N_{\text {vert }} \otimes \mathbb{C}\right), 0\right)$ :

$$
0 \rightarrow \Lambda^{0}\left(p_{T}^{*}\left(N_{\text {vert }} \otimes \mathbb{C}\right)\right) \stackrel{0}{\longrightarrow} \ldots \stackrel{0}{\longrightarrow} \Lambda^{k}\left(p_{T}^{*}\left(N_{\text {vert }} \otimes \mathbb{C}\right)\right) \rightarrow 0
$$

with noncompact support. If $a \in K_{\mathcal{G}}^{0}\left(T_{\text {vert }} X\right)$, then the complex

$$
a \otimes \Lambda\left(p_{T}^{*}\left(N_{\text {vert }} \otimes \mathbb{C}\right), 0\right)
$$

has compact support and defines an element of $K_{\mathcal{G}}^{0}\left(T_{\text {vert }} X\right)$. Then

$$
(d i)^{*} i_{!}(a)=a \cdot \Lambda\left(p_{T}^{*}\left(N_{\text {vert }} \otimes \mathbb{C}\right), 0\right)
$$

where di is the differential of the embedding $i$.

(vii) $i_{!}\left(x(d i)^{*} y\right)=i_{!}(x) \cdot y$, where $x \in K_{\mathcal{G}}^{0}\left(T_{\text {vert }} X\right)$ and $y \in K_{\mathcal{G}}^{0}\left(T_{\text {vert }} Y\right)$.

Proof. (i) This follows from the definition of $i_{!}$.

(ii) To simplify the argument, let us identify the tubular neighborhood with the normal bundle. Then $(j \circ i)$ ! is the composition

$$
K_{\mathcal{G}}^{0}\left(T_{\text {vert }} X\right) \stackrel{\varphi}{\longrightarrow} K_{\mathcal{G}}^{0}\left(T_{\text {vert }} N \oplus T_{\text {vert }} N_{\text {vert }}^{\prime \prime}\right) \rightarrow K_{\mathcal{G}}^{0}\left(T_{\text {vert }} Z\right),
$$

where $N_{v e r t}^{\prime}$ is the fiberwise normal bundle of $Y$ in $Z, N_{v e r t}^{\prime \prime}=\left.N_{v e r t}^{\prime}\right|_{X}$, and for the sum of tangent bundles to the vertical normal bundles $T_{\text {vert }} N \oplus T_{\text {vert }} N_{\text {vert }}^{\prime \prime}$ is considered in the same way as on page 21 , that is, as a complex bundle over $T_{\text {vert }} X$. On the other hand, $j_{!} \circ i_{\text {! }}$ represents the composition

$$
K_{\mathcal{G}}^{0}\left(T_{\text {vert }} X\right) \stackrel{\varphi}{\longrightarrow} K_{\mathcal{G}}^{0}\left(T_{\text {vert }} N\right) \rightarrow K_{\mathcal{G}}^{0}\left(T_{\text {vert }} Y\right) \stackrel{\varphi}{\longrightarrow} K_{\mathcal{G}}^{0}\left(T_{\text {vert }} N_{\text {vert }}^{\prime}\right) \rightarrow K_{\mathcal{G}}^{0}\left(T_{\text {vert }} Z\right) .
$$

By the properties of $\varphi$, the following diagram is commutative

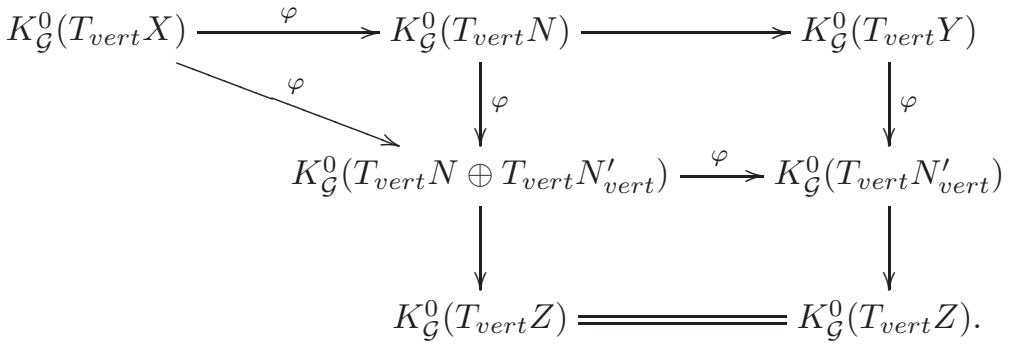

This completes the proof of (ii).

(iii) The morphism $q_{T}$ depends only on the homotopy class of the embeddings used to define it. The assertion thus follows from the homotopy invariance of $K$ theory.

(iv) In this case $N=X, W=Y, \Phi=i, i_{2}=\operatorname{Id}_{Y}$, and the formula is obvious.

(v) This follows from (ii).

(vi) By definition,

$(d i)^{*} \circ i_{!}=\left(d i_{1}\right)^{*} \circ\left(d i_{2}\right)^{*} \circ\left(d i_{2}\right)_{*} \circ\left(d \varphi^{-1}\right)^{*} \circ \psi^{*} \circ \varphi^{*}=\left(\psi \circ d \Phi^{-1} \circ d i_{1}\right)^{*} \circ \varphi$, 
where $i_{1}: X \rightarrow W, i_{2}: W \rightarrow Y$. Let $\left(n_{1}, t+n_{2}\right) \in T_{\text {vert }} N=p_{N}^{*}\left(T_{\text {vert }} X\right) \oplus$ $p_{N}^{*}\left(N_{\text {vert }}\right)$, where $n_{1}$ is the shift under the exponential mapping and $t+n_{2}$ is a vertical tangent vector to $W$. If $d \Phi\left(n_{1}, t+n_{2}\right)$ is in $T_{\text {vert }} X$, then $n_{1}=n_{2}=0$. Hence,

$$
d \Phi^{-1} d i_{1}(t)=(0, t+0), \quad \psi \circ d \Phi^{-1} \circ d i_{1}(t)=(t, 0+0) .
$$

Therefore, $\psi \circ d \Phi^{-1} \circ d i_{1}: T_{\text {vert }} X \rightarrow p_{T}^{*}\left(N_{\text {vert }} \oplus N_{\text {vert }}\right)$ is the embedding of the zero section. Since $\varphi(a)=a \cdot \Lambda\left(q_{T}^{*} p_{T}^{*}\left(N_{\text {vert }} \otimes \mathbb{C}\right), s_{p_{T}^{*}\left(N_{\text {vert }} \otimes \mathbb{C}\right)}\right)$, it follows that $(d i)^{*} \circ i_{!}(a)=a \cdot \Lambda\left(p_{T}^{*}\left(N_{\text {vert }} \otimes \mathbb{C}\right), 0\right)$.

(vii) The mapping $d i_{1} \circ q_{T} \circ \psi \circ d \Phi^{-1}: T_{\text {vert }} W \rightarrow T_{\text {vert }} W$ is homotopic to the identical mapping. Hence,

$$
\begin{aligned}
& i_{!}\left(x \cdot(d i)^{*} y\right)=\left(d i_{2}\right)_{*}\left(d \Phi^{-1}\right)^{*} \psi^{*} \varphi\left(x \cdot(d i)^{*} y\right)= \\
& =\left(d i_{2}\right)_{*}\left(d \Phi^{-1}\right)^{*} \psi^{*}\left[\left(q_{T}^{*}(x) \lambda_{p_{T}^{*}\left(N_{\text {vert }} \otimes \mathbb{C}\right)}\right)\left(q_{T}^{*}(d i)^{*} y\right)\right]= \\
& =\left(d i_{2}\right)_{*}[\left(d \Phi^{-1}\right)^{*} \psi^{*}\left(q_{T}^{*}(x) \lambda_{p_{T}^{*}\left(N_{\text {vert }} \otimes \mathbb{C}\right)}\right) \underbrace{\left(d \Phi^{-1}\right)^{*} \psi^{*} q_{T}^{*}\left(d i_{1}\right)^{*}}_{\text {Id }}\left(d i_{2}\right)^{*} y]= \\
& =\left[\left(d i_{2}\right)_{*}\left(d \Phi^{-1}\right)^{*} \psi^{*}\left(q_{T}^{*}(x) \lambda_{p_{T}^{*}\left(N_{\text {vert }} \otimes \mathbb{C}\right)}\right)\right]\left[\left(d i_{2}\right)_{*}\left(d i_{2}\right)^{*} y\right]=i_{!}(x) \cdot y .
\end{aligned}
$$

The proof is now complete.

We shall need also the following properties of the Gysin map. If $X=B$, the trivial longitudinally smooth $\mathcal{G}$-bundle, we shall identify $T_{\text {vert }} X=B$ and $T_{\text {vert }} \mathcal{V}=$ $\mathcal{V} \otimes \mathbb{C}$ for a real bundle $\mathcal{V} \rightarrow B$.

Theorem 5.4. Suppose that $\mathcal{V} \rightarrow B$ is a $\mathcal{G}$-equivariant real vector bundle and that $X=B$. Then the mapping

$$
i_{!}: K_{\mathcal{G}}^{0}(B)=K_{\mathcal{G}}^{0}\left(T X_{\text {vert }}\right) \rightarrow K_{\mathcal{G}}^{0}\left(T_{\text {vert }} \mathcal{V}\right)=K_{\mathcal{G}}^{0}(\mathcal{V} \otimes \mathbb{C})
$$

coincides with the Thom homomorphism $\varphi^{\mathcal{V} \otimes \mathbb{C}}$.

Proof. The assertion follows from the definition of $i_{\text {! }}$. More precisely, let $X=$ $B \hookrightarrow \mathcal{V}, N=\mathcal{V}$ be the zero section embedding. In the definition of the Thom isomorphism, $W$ can be chosen to be equal to the bundle $D_{1}$ of interiors of the balls of radius 1 in $\mathcal{V}$ with respect to an invariant metric. In this case, the diagram from the definition of the Gysin homomorphism 5.2 takes the following form

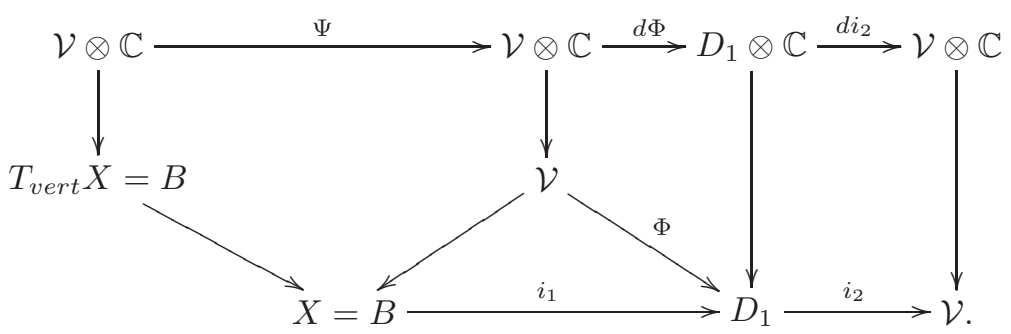

In our case $\Psi=\mathrm{Id}$ and $d i_{2} \circ d \Phi$ is homotopic to Id, since this map has the form $v \otimes z \mapsto(v \otimes z) /(1+|v \otimes z|)$. Hence, $i_{!}=\varphi$.

Theorem 5.5. Suppose that $\mathcal{V}^{\prime}$ and $\mathcal{V}^{\prime \prime}$ are $\mathcal{G}$-equivariant $\mathbb{R}$-vector bundles over $B$ and that $i: X \rightarrow \mathcal{V}^{\prime}$ is an embedding. Let $k: X \rightarrow \mathcal{V}^{\prime} \oplus \mathcal{V}^{\prime \prime}, k(x)=i(x)+0$. Let $\varphi$ be the Thom homomorphism of the complex bundle

$$
T_{\text {vert }}\left(\mathcal{V}^{\prime} \oplus \mathcal{V}^{\prime \prime}\right)=\mathcal{V}^{\prime} \otimes \mathbb{C} \oplus \mathcal{V}^{\prime \prime} \otimes \mathbb{C} \longrightarrow T_{\text {vert }} \mathcal{V}^{\prime}=\mathcal{V}^{\prime} \otimes \mathbb{C}
$$


Then the following diagram is commutative

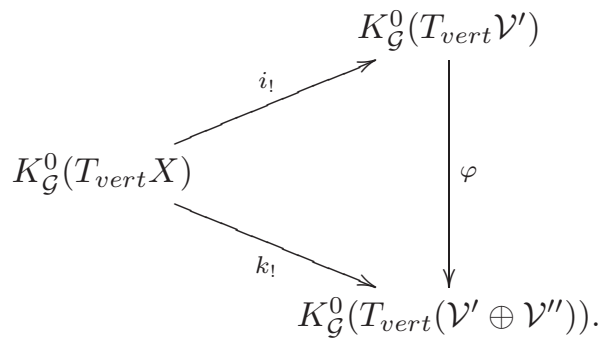

Proof. To prove the statement, let us consider the embedding $i: X \rightarrow \mathcal{V}^{\prime}$ and denote, as before, by $N_{\text {vert }}$ the fiberwise normal bundle and by $W$ the tubular neighborhood appearing in the definition of the Thom isomorphism associated to $i$. Then $N_{\text {vert }} \oplus \mathcal{V}^{\prime \prime}$ is a fiberwise normal bundle for the embedding $k$ with the tubular neighborhood $W \oplus D\left(\mathcal{V}^{\prime \prime}\right)$, where $D\left(\mathcal{V}^{\prime \prime}\right)$ is a ball bundle. If $a \in K_{\mathcal{G}}^{0}\left(T_{\text {vert }} X\right)$, then

$$
k_{!}(a)=\left(d i_{2} \oplus 1\right)_{*} \circ\left(d \Phi^{-1} \oplus 1\right)^{*} \circ(\psi \oplus 1)^{*} \circ \varphi^{N \oplus N \oplus \mathcal{V}^{\prime \prime} \oplus \mathcal{V}^{\prime \prime}}(a) .
$$

We have $\varphi^{N \oplus N \oplus \mathcal{V}^{\prime \prime} \oplus \mathcal{V}^{\prime \prime}}=\varphi^{N \oplus N} \circ \varphi^{\mathcal{V}^{\prime \prime} \oplus \mathcal{V}^{\prime \prime}}$, by Theorem 4.4. Since $a=a \cdot \underline{\mathbb{C}}$, where $\underline{\mathbb{C}}$ is the trivial line bundle, we obtain

$$
\begin{aligned}
k_{!}(a)=\left(d i_{2}\right)_{*} \circ\left(d \Phi^{-1}\right)^{*} \circ \Psi^{*} \circ \varphi^{N_{\text {vert }} \oplus N_{\text {vert }}}(a) \circ \varphi^{\mathcal{V}^{\prime \prime} \oplus \mathcal{V}^{\prime \prime}}(\underline{\mathbb{C}}) \\
\\
=i_{!}(a) \cdot \lambda_{T\left(\mathcal{V}^{\prime} \oplus \mathcal{V}^{\prime \prime}\right)_{\text {vert }}}=\varphi\left(i_{!}(a)\right) .
\end{aligned}
$$

The proof is now complete.

\section{The TOPOLOGiCAL INDEX}

We begin with a "fibered Mostow-Palais theorem" that will be useful in defining the index.

Theorem 6.1. Let $\pi_{X}: X \rightarrow B$ be a compact $\mathcal{G}$-fiber bundle. Then there exists a real $\mathcal{G}$-equivariant vector bundle $\mathcal{V} \rightarrow B$ and a fiberwise smooth $\mathcal{G}$-embedding $X \rightarrow \mathcal{V}$. After averaging one can assume that the action of $\mathcal{G}$ on $\mathcal{V}$ is orthogonal.

Proof. Fix $b \in B$ and let $U$ be an equivariant trivialization neighborhood of $b$ for both $X$ and $\mathcal{G}$. By the Mostow-Palais theorem, there exists a representation of $\mathcal{G}_{b}$ on a finite dimensional vector space $V_{b}$ and a smooth $\mathcal{G}_{b}$-equivariant embedding $i_{b}: X_{b} \rightarrow V_{b}$. This defines an embedding

$$
\psi: \pi_{X}^{-1}\left(U_{b}\right) \simeq U_{b} \times X_{b} \rightarrow U_{b} \times V_{b}
$$

which is $\mathcal{G}$-equivariant in an obvious sense.

We can cover $B$ with finitely many open sets $U_{b_{j}}$, as above, corresponding to the points $b_{j}, j=1, \ldots, N$. Denote by $V_{j}$ the corresponding representations and by $\psi_{j}$ the corresponding embeddings, as in Equation (24). Let $W:=\oplus V_{j}$. Also, Let $\phi_{j}$ be a partition of unity subordinated to the covering by $U_{j}=U_{b_{j}}$. We define then

$$
\Psi:=\oplus\left(\phi_{j} \circ \pi_{X}\right) \psi_{j}: X \rightarrow B \times W,
$$

which is a $\mathcal{G}$-equivariant embedding of $X$ into the trivial $\mathcal{G}$-equivariant vector bundle $\mathcal{V}:=B \times W$, as desired. 
Let us now turn to the definition of the topological index. Let $X \rightarrow B$ be a compact, longitudinally smooth $\mathcal{G}$-bundle. From Theorem 6.1 it follows that there exists an $\mathcal{G}$-equivariant real vector bundle $\mathcal{V} \rightarrow B$ and a fiberwise smooth $\mathcal{G}$-equivariant embedding $i: X \rightarrow \mathcal{V}$. We can assume that $\mathcal{V}$ is endowed with an orthogonal metric and that $\mathcal{G}$ preserves this metric. Thus, the Gysin homomorphism

$$
i_{!}: K_{\mathcal{G}}^{0}\left(T_{\text {vert }} X\right) \rightarrow K_{\mathcal{G}}^{0}\left(T_{\text {vert }} \mathcal{V}\right)=K_{\mathcal{G}}^{0}(\mathcal{V} \otimes \mathbb{C})
$$

is defined (see Section 4). Since $T_{\text {vert }} \mathcal{V}=\mathcal{V} \otimes \mathbb{C}$ is a complex vector bundle, we have the following Thom isomorphism (see Section 4):

$$
\varphi: K_{\mathcal{G}}^{0}(B) \stackrel{\sim}{\longrightarrow} K_{\mathcal{G}}^{0}\left(T_{\text {vert }} \mathcal{V}\right)
$$

Definition 6.2. The topological index is by definition the morphism:

$$
\operatorname{t}_{-i n d}^{X}: K_{\mathcal{G}}^{0}\left(T_{\text {vert }} X\right) \rightarrow K_{\mathcal{G}}^{0}(B), \quad \operatorname{t}_{-} \operatorname{ind}_{\mathcal{G}}^{X}:=\varphi^{-1} \circ i_{!} .
$$

The topological index satisfies the following properties.

Theorem 6.3. Let $X \rightarrow B$ be a longitudinally smooth bundle and

$$
\mathrm{t}_{\text {-ind }}^{X}: K_{\mathcal{G}}^{0}\left(T_{\text {vert }} X\right) \rightarrow K_{\mathcal{G}}^{0}(B)
$$

be its associated topological index. Then

(i) $\operatorname{tind}_{\mathcal{G}}^{X}$ does not depend on the choice of the $\mathcal{G}$-equivariant vector bundle $\mathcal{V}$ and on the embedding $i: X \rightarrow \mathcal{V}$.

(ii) $\mathrm{t}$-ind $\mathrm{G}_{\mathcal{G}}^{X}$ is a $K_{\mathcal{G}}^{0}(B)$-homomorphism.

(iii) If $X=B$, then the map

$$
\mathrm{t}-\operatorname{ind}_{\mathcal{G}}^{X}: K_{\mathcal{G}}^{0}(B)=K_{\mathcal{G}}^{0}\left(T_{\text {vert }} X\right) \rightarrow K_{\mathcal{G}}^{0}(B)
$$

coincides with $\operatorname{Id}_{K_{\mathcal{G}}^{0}(B)}$.

(iv) Suppose $X$ and $Y$ are compact longitudinally smooth $\mathcal{G}$-bundles, $i: X \rightarrow Y$ is a fiberwise $\mathcal{G}$-embedding. Then the diagram

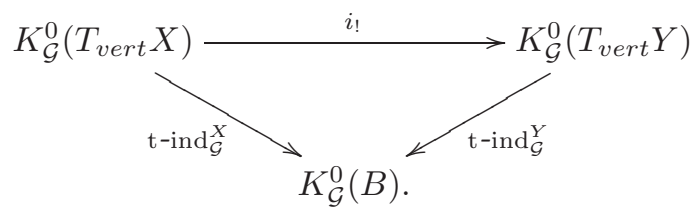

commutes.

Proof. To prove (i), let us consider two embeddings

$$
i_{1}: X \rightarrow \mathcal{V}^{\prime}, \quad i_{2}: X \rightarrow \mathcal{V}^{\prime \prime}
$$

into $\mathcal{G}$-equivariant vector bundles. Denote by $j=i_{1}+i_{2}$ the induced embedding $j: X \rightarrow \mathcal{V}^{\prime} \oplus \mathcal{V}^{\prime \prime}$. It is sufficient to show that $i_{1}$ and $j$ define the same topological index. Let us define a homotopy of $\mathcal{G}$-embeddings by the formula

$$
j_{s}(x)=i_{1}(x)+s \cdot i_{2}(x): X \rightarrow \mathcal{V}^{\prime} \oplus \mathcal{V}^{\prime \prime}, \quad 0 \leq s \leq 1
$$

Then, by Theorems 5.3(iii) and 5.5, the indices for $j$ and $j_{0}$ coincide. Let us show now that $j_{0}=i_{1}+0$ and $i_{1}$ define the same topological indexes. For this purpose 
consider the diagram

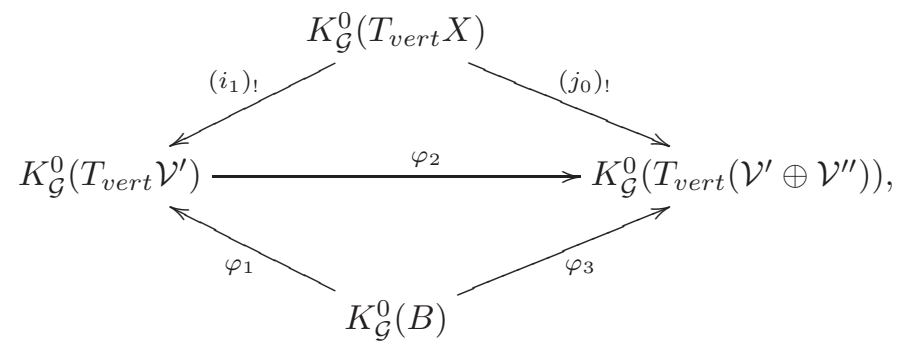

where $\varphi_{i}$ are the corresponding Thom homomorphisms. The upper triangle is commutative by Theorem 5.4.2, and the lower is commutative by Theorem 4.4. Hence $\varphi_{1}^{-1} \circ\left(i_{1}\right) !=\varphi_{3}^{-1} \circ\left(j_{0}\right)_{1}$ as desired.

(ii) follows from 4.2 and $5.3(\mathrm{i})$.

Property (iii) follows from the definition of the index and from 5.4.

To prove (iv), let us consider the diagram

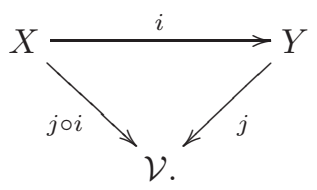

We now use 5.3(ii). This gives the commutative diagram

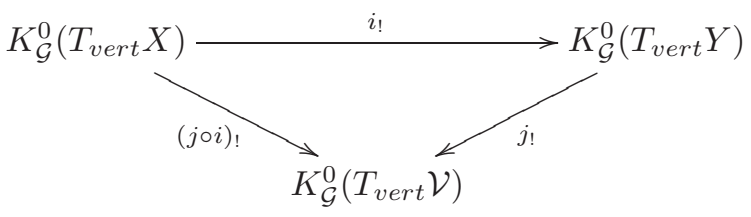

or

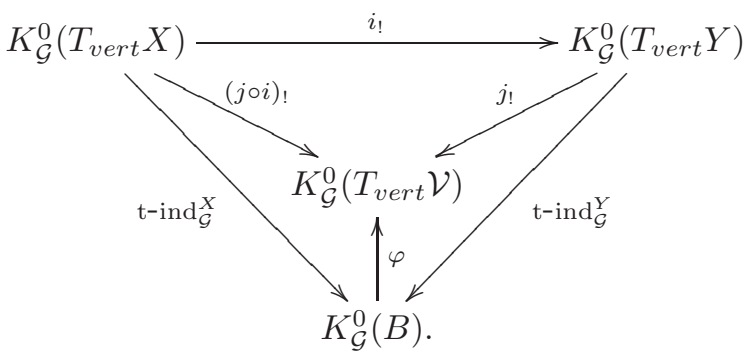

This completes the proof.

We now investigate the behavior of the topological index with respect to fiber products of bundles of compact groups.

Theorem 6.4. Let $\pi: P \rightarrow X$ be a principal right $\mathcal{H}$-bundle with a left action of $\mathcal{G}$ commuting with $\mathcal{H}$. Suppose $F$ is a longitudinally smooth $(\mathcal{G} \times \mathcal{H})$-bundle. Let us denote by $Y$ the space $P \times_{\mathcal{H}} F$. Let $j: X^{\prime} \rightarrow X$ and $k: F^{\prime} \rightarrow F$ be fiberwise $\mathcal{G}$ and $(\mathcal{G} \times \mathcal{H})$-embeddings, respectively. Let $\pi^{\prime}: P^{\prime} \rightarrow X^{\prime}$ be the principal $\mathcal{H}$-bundle induced by $j$ on $X^{\prime}$. Assume that $Y^{\prime}:=P^{\prime} \times_{\mathcal{H}} F^{\prime}$. The embeddings $j$ and $k$ induce 
$\mathcal{G}$-embedding $j * k: Y^{\prime} \rightarrow Y$. Then the diagram

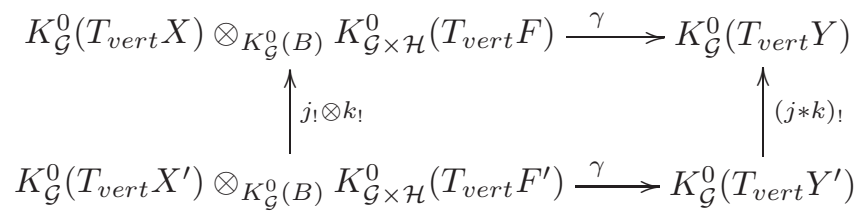

is commutative.

Let us remark that there in the statement of this theorem there is no compactness assumption on $X, X^{\prime}, F$, and $F^{\prime}$, since there is no compactness assumption in the definition of the Gysin homomorphism. This is unlike in the definition of the topological index where we start with a compact $\mathcal{G}$-bundle $X \rightarrow B$.

Proof. Let us use the definition of $\gamma$ :

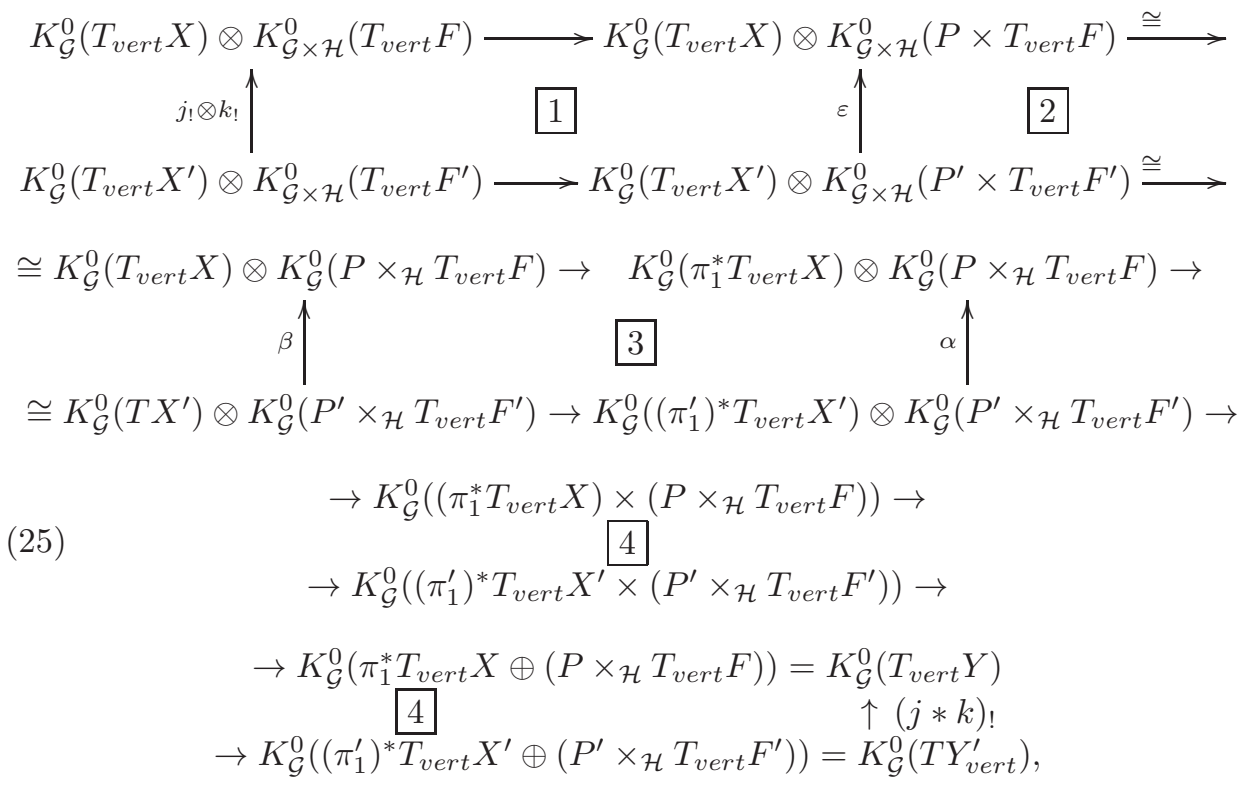

where projections $\pi_{1}: Y=P \times_{\mathcal{H}} F \rightarrow X$ and $\pi_{1}^{\prime}: Y^{\prime}=P \times_{\mathcal{H}} F^{\prime} \rightarrow X^{\prime}$ are defined as above. Here we use the isomorphism $K_{\mathcal{G} \times \mathcal{H}}^{0}(P \times W) \cong K_{\mathcal{G}}^{0}\left(P \times_{\mathcal{H}} W\right)$ for a free $\mathcal{H}$-bundle $P$ (see Theorem 2.6). Let us remind the diagram, which was used for the definition of the Gysin homomorphism of an embedding $j: X^{\prime} \rightarrow X$ :

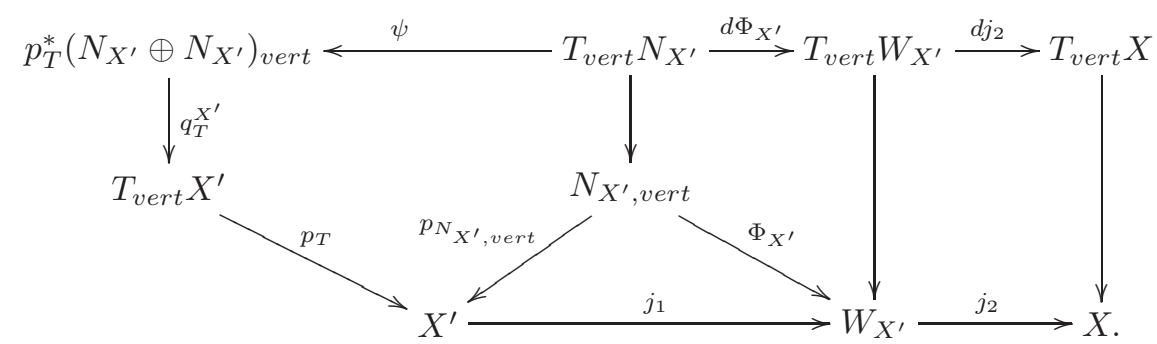

From the similar diagrams for $k$ ! and $(j * k)$ ! and the explicit form of these maps, it follows that the square 4 in (25) is commutative if, and only if, $\alpha$ has the following 
form:

$$
\begin{aligned}
& \alpha(\sigma \otimes \rho)=\left(\pi_{1}^{*}\right)\left\{\left(d j_{2}\right)_{*}\left(d \Phi_{X^{\prime}}^{-1}\right)^{*} \psi_{X^{\prime}}^{*}\right\} \circ \varphi^{S}(\sigma) \otimes \\
& \quad \otimes\left(\pi^{*} j_{2} \times_{\mathcal{H}} d k_{2}\right)_{*}\left(\left(\pi^{*} \Phi_{X^{\prime}} \times_{\mathcal{H}} d \Phi_{F^{\prime}}\right)^{-1}\right)^{*}\left(1 \times_{\mathcal{H}} \psi_{F^{\prime}}\right)^{*} \varphi^{R}(\rho),
\end{aligned}
$$

where $S$ and $T$ are bundles of the form

$$
\begin{array}{cccc} 
& \pi_{1}^{*}\left(\left(p_{T}^{X^{\prime}}\right)^{*}\left\{N_{X^{\prime}} \oplus N_{X^{\prime}}\right\}\right) & & \pi^{*} N_{X^{\prime}} \times \mathcal{H}\left(p_{T}^{F^{\prime}}\right)^{*}\left(N_{F^{\prime}} \oplus N_{F^{\prime}}\right) \\
S: \quad & \downarrow\left(\pi_{1}^{\prime}\right)^{*} q_{T}^{X^{\prime}} & R: & \downarrow \\
& \left(\pi_{1}^{\prime}\right)^{*}\left(T_{\text {vert }} X^{\prime}\right), & & \pi^{*} X^{\prime} \times_{\mathcal{H}} T_{\text {vert }} F^{\prime}=P^{\prime} \times_{\mathcal{H}} T_{\text {vert }} F^{\prime} .
\end{array}
$$

Hence the square 3 in (25) is commutative iff the homomorphism $\beta$ has the form

$$
\begin{aligned}
& \beta(\tau \otimes \rho)=j_{!}(\tau) \otimes \\
& \otimes\left(\pi^{*} j_{2} \times_{\mathcal{H}} d k_{2}\right)_{*}\left(\left(\pi^{*} \Phi_{X^{\prime}} \times_{\mathcal{H}} d \Phi_{F^{\prime}}\right)^{-1}\right)^{*}\left(1 \times_{\mathcal{H}} \psi_{F^{\prime}}\right)^{*} \varphi^{R}(\rho), \\
& \tau \in K_{\mathcal{G}}^{0}\left(T X^{\prime}\right), \quad \rho \in K_{\mathcal{G}}^{0}\left(P^{\prime} \times_{\mathcal{H}} T F^{\prime}\right) .
\end{aligned}
$$

In turn, the square 2 in (25) is commutative iff the homomorphism $\varepsilon$ has the form

$$
\begin{gathered}
\varepsilon(\tau \otimes \delta)=j_{!}(\tau) \otimes \\
\otimes\left(\pi^{*} j_{2} \times_{\mathcal{H}} d k_{2}\right)_{*}\left(\left(\pi^{*} \Phi_{X^{\prime}} \times_{\mathcal{H}} d \Phi_{F^{\prime}}\right)^{-1}\right)^{*}\left(1 \times_{\mathcal{H}} \psi_{F^{\prime}}\right)^{*} \varphi_{\mathbb{C}}^{\widetilde{R}}(\delta), \\
\tau \in K_{\mathcal{G}}^{0}\left(T X^{\prime}\right), \quad \delta \in K_{G \times \mathcal{H}}^{0}\left(P^{\prime} \times T F^{\prime}\right),
\end{gathered}
$$

where $\widetilde{R}$ is the following bundle:

$$
\begin{aligned}
\pi^{*} N_{X^{\prime}} & \times\left(p_{T}^{F^{\prime}}\right)^{*}\left(N_{F^{\prime}} \oplus N_{F^{\prime}}\right) \\
\widetilde{R}: \quad \downarrow & \left(\pi^{\prime}\right)^{*}\left(p_{N}\right) \times q_{T}^{F^{\prime}} \\
P^{\prime} & \times T F^{\prime} .
\end{aligned}
$$

Suppose $\delta=[\mathbb{\mathbb { C }}] \widehat{\otimes} \omega$, where $[\underline{\mathbb{C}}] \in K_{\mathcal{G} \times \mathcal{H}}^{0}\left(P^{\prime}\right), \underline{\mathbb{C}}$ is the one-dimensional trivial bundle and $\omega \in K_{\mathcal{G} \times \mathcal{H}}^{0}\left(T F^{\prime}\right)$. Then

$$
\begin{aligned}
\varepsilon(\tau \otimes \delta) & =j_{!}(\tau) \otimes\left\{\pi^{*}\left(j_{2}\right)_{*}\left(\Phi_{X^{\prime}}^{-1}\right)^{*}[\mathbb{C}] \widehat{\otimes} k_{!}(\omega)\right\}= \\
& =j_{!}(\tau) \otimes\left\{[\mathbb{C}] \widehat{\otimes} k_{!}(\omega)\right\} .
\end{aligned}
$$

Since the map $K_{\mathcal{G} \times \mathcal{H}}^{0}(T F) \rightarrow K_{\mathcal{G} \times \mathcal{H}}^{0}(P \times T F)$ (as well as the lower line in (25)) has the form $\omega \mapsto[\mathbb{C}] \widehat{\otimes} \omega$, we have proved the commutativity of 1 in (25).

From this theorem we obtain the following corollary.

Corollary 6.5. Let $M$ be a compact smooth $H$-manifold, let $\mathcal{H}=B \times H$, and let $P$ be a principal longitudinally smooth $\mathcal{H}$-bundle over $X$ carrying also an action of $\mathcal{G}$ commuting with the action of $\mathcal{H}$. Also, let $X \rightarrow B$ be a compact longitudinally smooth $\mathcal{G}$-bundle. Let $Y:=P \times_{H} M \rightarrow X$ be associated longitudinally smooth $\mathcal{G}$-bundle. Taking $F=B \times M$, we define $T_{M} Y:=T_{F} Y$. Then $T_{M} Y$ is a $\mathcal{G}$ invariant real subbundle of $T_{\text {vert }} Y$ and $T_{M} Y=P \times_{H} T M$. Let $j: X^{\prime} \rightarrow X$ be a fiberwise $\mathcal{G}$-equivariant embedding and let $k: M^{\prime} \rightarrow M$ be an $H$-embedding. Denote by $\pi^{\prime}: P^{\prime} \rightarrow X^{\prime}$ the principal $\mathcal{H}$-bundle induced by $j$ on $X^{\prime}$ and assume 
that $Y^{\prime}:=P^{\prime} \times_{H} M^{\prime}$. The embeddings $j$ and $k$ induce $\mathcal{G}$-embedding $j * k: Y^{\prime} \rightarrow Y$. Then the diagram

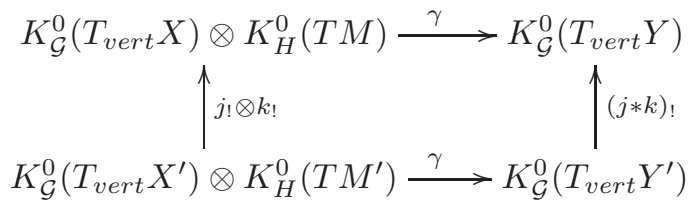

is commutative.

\section{REFERENCES}

[1] M. Atiyah and G. Segal, Twisted K-theory, e-print math.KT/0407054.

[2] M. F. Atiyah, K-theory, W.A. Benjamin, Inc., New York - Amsterdam, 1967.

[3] — Bott periodicity and the index of elliptic operators, Quart. J. Math. Oxford 19 (1968), 113-140.

[4] M. F. Atiyah and I. M. Singer, The index of elliptic operators. I, Ann. of Math. (2) 87 (1968), $484-530$.

[5] C. Carvalho, $C^{*}$-algebraic $K$-theory and index theory, PhD dissertation, Oxford University, Department of Math., 2003, (to appear in K-Theory).

[6] A. Connes, An analogue of the Thom isomorphism for crossed products of a $C^{*}$-algebra by an action of $\mathbb{R}$, Adv. Math. 39 (1981), 31-55.

[7] Th. Fack and G. Skandalis, Connes' analogue of the Thom isomorphism for the Kasparov groups, Invent. Math. 64 (1981), 7-14.

[8] D. S. Freed, Twisted $k$-theory and loop groups, International Congress of Mathematicians (2002, Beijing), Higher Education Press, Beijing, 2002, pp. 419-430.

[9] D. S. Freed, M. J. Hopkins, and C. Teleman, Twisted equivariant K-theory with complex coefficients, E-print, math.AT/0206257.

[10] T. Friedrich, Vorlesungen über K-Theorie, Teubner, Leipzig, 1987.

[11] M. Hilum and G. Skandalis, Invariance par homotopie de la signature à coefficients dans un fibré presque plat, J. Rene Angew. Math 423 (1992), 73-99.

[12] V. Mathai, R. B. Melrose, and I. M. Singer, The index of projective families of elliptic operators, E-print, math.DG/0206002.

[13] V. Nistor, An index theorem for gauge-invariant families: The case of solvable groups, Acta Math. Hungar. 99 (2003), no. 1-2, 155-183.

[14] V. Nistor and E. Troitsky, An index theorem for gauge-invariant families: The case of compact groups, (tentative title, work in progress).

[15] - An index for gauge-invariant operators and the Dixmier-Douady invariant., Trans. Am. Math. Soc. 356 (2004), no. 1, 185-218.

[16] E. V. Troitsky, "Twice" equivariant $C^{*}$-index theorem and the index theorem for families, Acta. Appl. Math. 68 (2001), 39-70.

Department of Mathematics, Pennsylvania State University

E-mail address: nistor@math.psu.edu

URL: http://www.math.psu.edu/nistor

Dept. of Mech. and Math., Moscow State University, 119899 Moscow, Russia

E-mail address: troitsky@mech.math.msu.su

URL: http://mech.math.msu.su/ ${ }^{\sim}$ troitsky 